ARTICLE

\title{
UBE4B, a microRNA-9 target gene, promotes autophagy-mediated Tau degradation
}

\author{
Manivannan Subramanian ${ }^{1,2,7}$, Seung Jae Hyeon ${ }^{3,7}$, Tanuza Das ${ }^{4}$, Yoon Seok Suh ${ }^{1}$, Yun Kyung Kim (D) ${ }^{2}$,
} Jeong-Soo Lee (iD ${ }^{1,2}$, Eun Joo Song (10 ${ }^{5 凶}$, Hoon Ryu ${ }^{3 凶}$ \& Kweon Yu (1) ${ }^{1,2,6 凶}$

The formation of hyperphosphorylated intracellular Tau tangles in the brain is a hallmark of Alzheimer's disease (AD). Tau hyperphosphorylation destabilizes microtubules, promoting neurodegeneration in $A D$ patients. To identify suppressors of tau-mediated $A D$, we perform a screen using a microRNA (miR) library in Drosophila and identify the miR-9 family as suppressors of human tau overexpression phenotypes. CG11070, a miR-9a target gene, and its mammalian orthologue UBE4B, an E3/E4 ubiquitin ligase, alleviate eye neurodegeneration, synaptic bouton defects, and crawling phenotypes in Drosophila human tau overexpression models. Total and phosphorylated Tau levels also decrease upon CG11070 or UBE4B overexpression. In mammalian neuroblastoma cells, overexpression of UBE4B and STUB1, which encodes the E3 ligase CHIP, increases the ubiquitination and degradation of Tau. In the Tau-BiFC mouse model, UBE4B and STUB1 overexpression also increase oligomeric Tau degradation. Inhibitor assays of the autophagy and proteasome systems reveal that the autophagy-lysosome system is the major pathway for Tau degradation in this context. These results demonstrate that UBE4B, a miR-9 target gene, promotes autophagy-mediated Tau degradation together with STUB1, and is thus an innovative therapeutic approach for AD.

\footnotetext{
${ }^{1}$ Metabolism and Neurophysiology Research Group, KRIBB, Daejeon, Korea. ${ }^{2}$ Convergence Research Center of Dementia, KIST, Seoul, Korea. ${ }^{3}$ Center for Neuroscience, Brain Science Institute, KIST, Seoul, Korea. ${ }^{4}$ Biomedical Research Institute, KIST, Seoul, Korea. ${ }^{5}$ Graduate School of Pharmaceutical Sciences and College of Pharmacy, Ewha Womans University, Seoul, Korea. ${ }^{6}$ Department of Functional Genomics, UST, Daejeon, Korea. ${ }^{7}$ These authors contributed

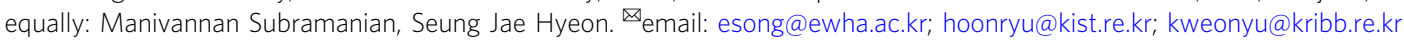


lzheimer's disease (AD) is one of the most common agerelated neurodegenerative diseases, and includes the pathological hallmarks of extracellular amyloid plaques by abnormally folded amyloid- $\beta 42(\mathrm{~A} \beta-42)$ and intracellular neurofibrillary tangles (NFTs) by Tau hyperphosphorylation in the brain $^{1,2}$. Tau is an essential soluble intracellular protein that associates with and stabilizes axon microtubules ${ }^{3}$. Hyperphosphorylation disrupts the physiological function of Tau, resulting in microtubule destabilization and formation of intracellular NFTs, potentiating neurodegeneration and memory impairment in $\mathrm{AD}$ patients. Neurodegenerative disorders with Tau inclusions are referred to as tauopathies ${ }^{4}$.

Drosophila melanogaster has emerged as an important model system for investigating the pathology of tauopathies at the cellular and molecular levels. Drosophila tauopathy AD models exhibit visible phenotypes such as brain vacuole formation, neuromuscular junction defects, larval and adult locomotor defects, impairment of learning and memory, and reduced lifespan ${ }^{5,6}$. Ectopic expression of human tau in Drosophila eyes induces a rough eye phenotype, which is widely used to screen for modifiers of Tau pathology ${ }^{7}$. Similarly, tissue-specific ectopic expression of human tau or mutant tau in the Drosophila brain or mushroom body induces neurodegeneration, manifesting in phenotypes such as locomotor and cognitive impairment ${ }^{8}$. The pathogenic mechanisms of Tau are similar in humans and Drosophila, ${ }^{9,}$.

MicroRNAs (miRNAs) are non-coding RNAs comprised of 19 to 25 nucleotides, which suppress target mRNAs by pairing to complementary sequences in the 3' UTRs of target genes, impairing translation and in some cases mRNA stability 11,12 . miRNAs are implicated in diverse brain functions including development, cognition, and synaptic plasticity ${ }^{13}$. Dysregulation of miRNAs can be detrimental, and is associated with several human diseases ranging from metabolic and inflammatory disease to neoplasia ${ }^{14-16}$. miRNA expression-profiling studies have identified that multiple miRNAs are dysregulated in the brains of $\mathrm{AD}$ patients, but the functional implications of these changes remain unclear ${ }^{17}$.

The ubiquitin-proteasome system (UPS) and autophagylysosome system (ALS) are the primary protein degradation pathways in eukaryotic cells ${ }^{18}$. Proteins that undergo proteasomal degradation are polyubiquitinated at Lys 48 by ubiquitin ligases and targeted for $26 \mathrm{~S}$ proteasome complex degradation and proteins poly-ubiquitinated at Lys63 are degraded by the ALS. Interestingly, proteostasis is disrupted in $\mathrm{AD}$ brains ${ }^{19}$. A prior study reported that Tau phosphorylation induces ubiquitination and subsequent degradation by the UPS ${ }^{20}$. STUB1 (STIP1 homology and U-Box containing protein1) is an E3 ubiquitin ligase that ubiquitinates phosphorylated Tau for proteasomal degradation in vitro ${ }^{21}$ and has also been implicated in the clearance of truncated Tau by the ALS 22 .

In the present study, we report the results of a Drosophila miRNA library screening, which identified the evolutionarily conserved miR-9 and its target CG11070 as strong modifiers of the neurodegenerative Drosophila rough eye phenotype induced by human tau overexpression. Further, Drosophila CG11070 and its mammalian orthologue $U B E 4 B$ (Ubiquitin conjugation $\mathrm{E} 4 \mathrm{~B}$ ) alleviated the neurodegenerative phenotypes induced by neuronal human tau overexpression in Drosophila. In addition, total and phosphorylated Tau in aged tau-overexpressing flies was decreased by $C G 11070$ or mammalian $U B E 4 B$ overexpression. In mammalian neuroblastoma cells and a mouse tau overexpression model, UBE4B, and STUB1 E3 ligases ubiquitinated Tau proteins and induced autophagy-mediated Tau degradation. These findings suggest that CG11070/UBE4B is a E4 ubiquitin ligase that regulates Tau degradation via the ALS, and is a putative therapeutic target for the treatment of tauopathies such as AD.

\section{Results}

Identification of $m i R-9$ as a modifier of $h T a u$ in Drosophila by genome-wide miRNA screening. The overexpression of human tau (hTau) in Drosophila eyes using the eye-specific GMR-GAL4 promoter induces eye neurodegeneration, which is grossly characterized by decreased eye size in the rough eye phenotype ${ }^{7}$. This phenotype was used to screen 131 UAS-miRNA lines covering 144 Drosophila miRNAs ${ }^{23}$. We analyzed the eye morphology of each line, quantitatively measured eye sizes, and arranged the library in the order of increasing eye size (Fig. 1a, Supplementary Fig. 1a, b). Eye-specific overexpression of many miRNAs in hTau flies $(G M R>h T a u)$ affected the eye size. Quantification of eye sizes was plotted in a volcano plot by the ratio of eye size to $p$-value of miRNA-overexpressing lines relative to the control GMR $>$ hTau line (Fig. 1b). The most significant reductions in eye sizes were induced by the overexpression of $m i R-9 a, m i R-9 b$, and $m i R-9 c$ (Fig. 1c, d), which are members of the evolutionarily conserved $m i R-9$ family (Fig. 1e). The reduced eye size of $m i R-9 a, m i R-9 b$ or $m i R-9 c$ in the absence of $h$ Tau expression (Fig. 1c, d, and Supplementary Fig. 1) may be due to the involvement of these miRNAs during eye development where these miRNAs may regulate other target genes and affect morphology and development of eye sizes. However, when these miRNAs were expressed in the presence of $h T a u$, the reduction of eye size was enhanced drastically. This indicated an important regulatory role for the miR-9 family in Drosophila Tau toxicity. We focused on $m i R-9 a$ for subsequent studies, as $m i R-9 a$ has $100 \%$ homology to mammalian miR-9 (Fig. 1e).

Modulation of hTau by CG11070, a $m i R-9 a$ target identified by secondary screening. Because $m i R-9 a$ was identified as a modifier of Tau toxicity, we searched $m i R-9 a$ target genes using three separate microRNA target prediction programs, TargetScan (www.targetscan.org), miRanda (www.microRNA.org), and miRbase (www.mirbase.org), detecting $34 \mathrm{miR}-9 a$ targets common to all three platforms (Supplementary Fig. 2a). We then screened the 34 putative target genes against the eye phenotype of $h T a u$ overexpression $(G M R>h T a u)$ using stably expressed RNAi knockdown in the GMR $>h$ Tau background, and arranged the RNAi lines in order of increasing eye size (Fig. 2a, Supplementary Fig. 2b, c). Volcano plot analysis was conducted by the ratio of eye size to $p$-value of the 34 RNAi lines relative to the control GMR > hTau flies. CG11070-RNAi flies exhibited the most prominent reduction in eye size (Fig. 2b, Supplementary Fig. 2c). The eye size of CG11070-RNAi flies in the GMR > hTau background $(G M R>h T a u+C G 11070-R N A i)$ was significantly reduced relative to the eye size of control GMR $>$ hTau flies (Fig. 2c, d).

Because microRNAs bind to $3^{\prime}$-UTR region of target mRNAs to suppress translation and/or mRNA stability, we assessed binding of $m i R-9 a$ to CG11070 using a miRNA-mRNA pulldown assay ${ }^{23}$. Similar to the known miR-9a/miR-9 targets senseless $^{24}$ and $s N P F R 1^{23}$, we found that $m i R-9 a$ bound to and enriched CG11070 mRNA in Drosophila S2 cells compared to its control scrambled miRNA (Fig. 2e). Consistently, eye-specific overexpression of $m i R-9 a$ with GMR-GAL4 flies decreased CG11070 mRNA levels (Fig. 2f). Taken together, these findings suggested that CG11070 was a legitimate target of miR-9a.

The overexpression of Drosophila CG11070 and its mammalian orthologue UBE4B alleviated hTau phenotypes in Drosophila. CG11070 and UBE4B proteins had 26\% amino acid identities and similar functional domains (Supplementary Fig. 3a). Interestingly, the $3^{\prime}$-UTR region of $U B E 4 B$ mRNA also contained miR-9 binding sequences, similar to the $3^{\prime}$-UTR region of CG11070 mRNA (Supplementary Fig. 3b). In addition to $m i R-9 a, U B E 4 B$ is 
a

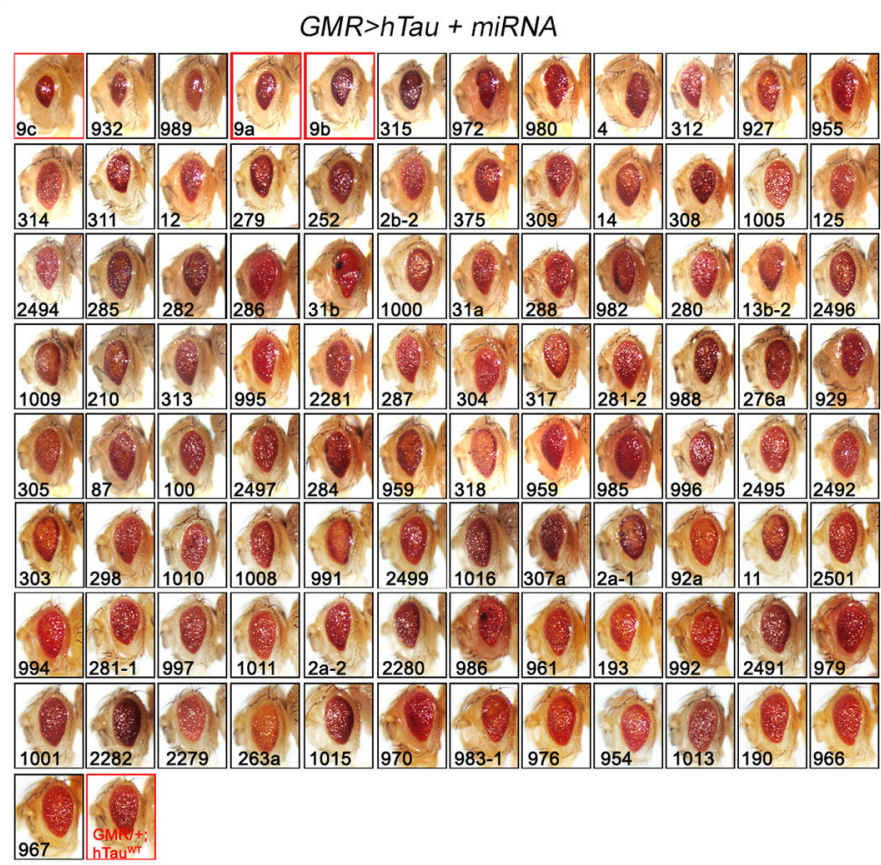

b

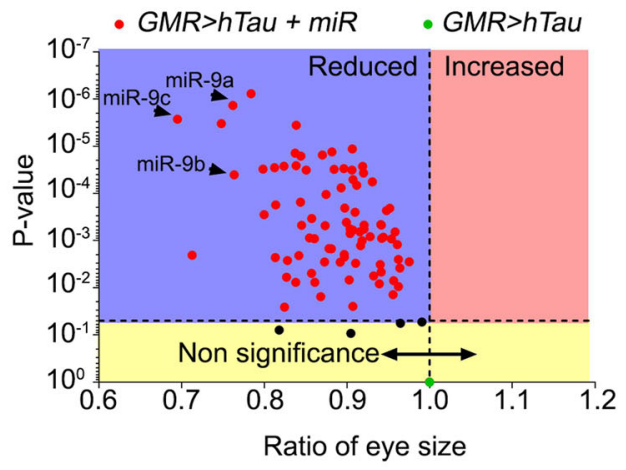

d

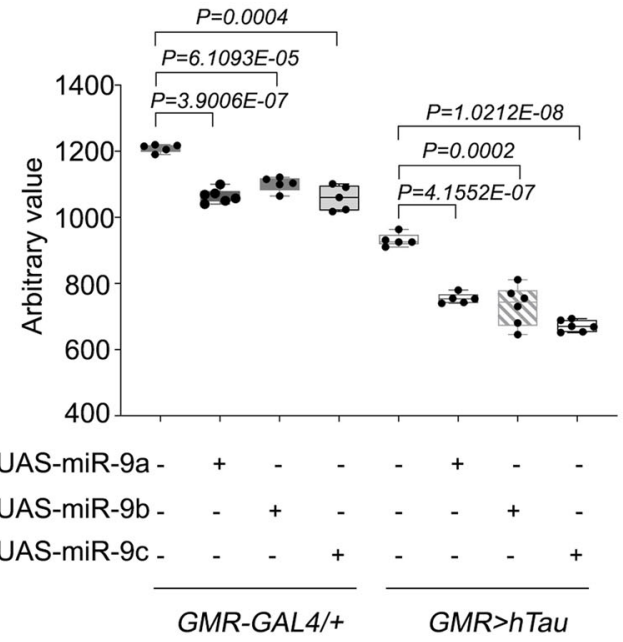
dme-miR-9a-5p UCUUUGGUUAUCUAGCUGUAUGA hsa-miR-9-5p UCUUUGGUUAUCUAGCUGUAUGA mmu-miR-9-5p UCUUUGGUUAUCUAGCUGUAUGA
dme-miR-9b-5p UCUUUGGUGAUUUUAGCUGUAUG
hsa-miR-9-5p UCUUUGGUUAUC---UAGCUGUAUGA
mmu-miR-9-5p UCUUUGGUUAUC---UAGCUGUAUGA
dme-miR_9c-5p UCUUUGGUAUUCUAGCUGUA--GA hsa-miR-9-5p UCUUUGGUUAUC---UAGCUGUAUGA mmu-miR-9-5p UCUUUGGUUAUCUAGCUGUAUGA

also regulated by $m i R-26, m i R-148 / m i R-152$, and $m i R-15 / 16 / 195 /$ 424/497. However, CG11070 is regulated by $m i R-9 a$ only in Drosophila. Therefore, CG11070/UBE4B was chosen for further analysis due to similarity in regulation by $m i R-9$. Because the knockdown of CG11070 in GMR > hTau flies decreased eye size relative to control GMR > hTau flies, we determined if the overexpression of CG11070 and its mammalian orthologue UBE4B could alleviate $h \mathrm{Tau}$ phenotypes in GMR $>h T a u$ flies.

In GMR > hTau flies, the overexpression of CG11070 (GMR > $h T a u+G C 11070)$ and UBE4B $(G M R>h T a u+U B E 4 B)$ increased 
Fig. 1 Genome-wide Drosophila miRNA library screening identified miR-9 family miRNAs as modifiers of $\boldsymbol{h T a u}$ in Drosophila eyes. a Screening of the miRNA library in GMR > hTau Drosophila eyes revealed significant phenotypic enhancement, as indicated by decreased eye size, in flies overexpressing miR9 family miRNAs compared with GMR > hTau control flies. Eye sizes were arranged from smallest to largest. b Volcano plot of mean eye sizes in flies expressing various miRNAs in the GMR > hTau background versus their respective $p$-values derived from a one-way analysis of variance followed by pairwise $t$-tests and a Bonferroni correction for multiple comparisons. All points above the dotted line can be considered significant. This analysis identified that flies overexpressing miR-9 family miRNAs exhibited a severe Tau toxicity phenotype, as indicated by decreased eye size. $N=3$ biologically independent experiments. c, d The overexpression of miR-9a, miR-9b, or miR-9c in GMR $>h T a u$ Drosophila eyes significantly reduced eye sizes relative to $G M R>h T a u$ Drosophila. $N=5$ biologically independent experiments. Data are presented as the mean \pm s.e.m. Statistical significance was determined with a two-tailed Student's $t$-test. In the box plots the whiskers represent the 5th to 95th percentile range. e Alignment of mature Drosophila miR-9a, miR-9b, and miR-9c sequences with human and murine miR-9 sequences identified that miR-9a had $100 \%$ identity with mammalian miR-9 sequences. Statistical source data.

eye size, alleviating the rough eye neurodegenerative phenotype (Fig. 3a, b). Neuron-specific ectopic hTau expression (Elav > hTau) decreased Drosophila larva locomotion, which was alleviated by $C G 11070$ and $U B E 4 B$ overexpression (Fig. 3c, d). The bouton number of neuromuscular junctions (NMJ) in Drosophila larvae is directly correlated with locomotion, and is decreased in Drosophila AD models ${ }^{25,26}$. Neuronal $h T a u$-expressing larvae exhibited significantly reduced bouton numbers relative to the control, which was alleviated by the overexpression of CG11070 and UBE4B (Fig. 3e, f).

The knockdown of miR-9a by miR-9a-sponge (SP) showed no change in the eye sizes when compared with GMR $>h T a u$ flies (Supplementary Fig. 4a, b). However, the knockdown by $m i R-9 a-$ $S P$ in neurons of Elav $>$ hTau rescued the larval locomotion phenotype similar to the overexpression of CG11070 and $h U B E 4 B$ (Supplementary Fig. 4c, d). Similarly, the knockdown by $m i R-9 a-$ $S P$ in neurons also rescued NMJ bouton numbers when compared with the overexpression of CG11070 and $h U B E 4 B$ in neurons (Supplementary Fig. 4e, f). This data also confirmed that $m i R-9 a$ and CG11070/UBE4B forms a common axis involved in regulating Tau toxicity in Drosophila.

Because the overexpression of CG11070 and UBE4B alleviated $h T a u$ phenotypes, we further examined whether the overexpression of these genes affected Tau degradation. We performed western blots on 30-day-old fly heads with eye-specific hTau expression $(G M R>h T a u)$, and identified that total hTau protein was significantly decreased by the overexpression of CG11070 $(G M R>h T a u+G C 11070)$ and UBE4B $(G M R>h T a u+U B E 4 B)$ (Fig. 3g, h). In addition, phosphorylated Tau, detected by AT8 (pS202/T205) antibody, was also significantly decreased by the overexpression of CG11070 and UBE4B (Fig. 3g, i). Similarly, other Tau phosphorylated forms, detected by AT180 (p-T231) and PHF-1 (p-S396/S404) antibodies, were also reduced in these genotypes (Supplementary Fig. 3c-e). Taken together, these findings suggested that the overexpression of either Drosophila CG11070 or human UBE4B rescued both larval locomotion/NMJ defects and adult eye phenotypes in hTau-overexpressing Drosophila by increasing Tau degradation.

Ubiquitination and degradation of Tau by UBE4B and STUB1 in mammalian neuroblastoma cells. To investigate the mechanism by which UBE4B degraded Tau, we first determined if UBE4B affected Tau ubiquitination in SH-SY5Y neuroblastoma cells (Fig. 4a). Previous studies have demonstrated that UBE4B has E4 ubiquitin ligase activity, and that Tau is ubiquitinated by STUB1 E3 ligase $21,27,28$. STUB1 was chosen due to its role in ubiquitination of Tau proteins and is not regulated by $m i R-9$. To confirm the effect of STUB1 on Tau ubiquitination, we evaluated ubiquitination of Tau by STUB1, and found that STUB1 overexpression alone did not affect Tau ubiquitination (Fig. 4a, lane 3). Similarly, $U B E 4 B$ overexpression alone did not significantly affect Tau ubiquitination (Fig. 4a, lane 4). However, UBE4B and
STUB1 co-expression significantly increased Tau ubiquitination (Fig. 4a, lane 5 compared with lane 3 and 4). Subsequently, we determined that STUB1 activity was important for UBE4Bmediated Tau ubiquitination, as co-expression with a dominantnegative mutant $\left(S T U B 1^{H 260 Q}\right)$ failed to enhance Tau ubiquitination (Fig. 4a, lane 6). These results suggested that UBE4B and STUB1 co-regulated Tau ubiquitination.

Due to the essential regulatory roles of these ubiquitin ligases in protein degradation, we examined UBE4B and STUB1mediated Tau degradation. By inhibiting protein synthesis with cycloheximide (CHX), we observed that Tau degradation was increased by STUB1 (Fig. 4b, lanes $\mathrm{f}-\mathrm{i}$, Fig. 4c) and UBE4B overexpression (Fig. 4b, lanes 1-4 and Fig. 4c), and was further increased by co-expression of $S T U B 1$ and $U B E 4 B$ (Fig. $4 \mathrm{~b}$, lanes $6-9$ relative to lanes $1-4$, Fig. $4 c$ ). Because $U B E 4 B$ overexpression alone degraded Tau (Fig. 4b, c), we knocked down endogenous STUB1 with siRNA (Supplementary Fig. 5a) and examined UBE4B-mediated Tau degradation (Supplementary Fig. 5b, c). Interestingly, $U B E 4 B$ overexpression did not affect Tau degradation when $S T U B 1$ was knocked down (Supplementary Fig. 5b, lanes 6-9 compared with lanes 1-4). Similarly, the knockdown of STUB1 showed no change in Tau degradation when compared with the siControl (Supplementary Fig. 5c). Collectively, these results indicated that UBE4B was a critical factor that enhanced the ubiquitination activity of STUB1 to ubiquitinate and degrade Tau. To evaluate the biochemical interactions of UBE4B with STUB1 and Tau, we performed immunoprecipitation analyses. When Tau was co-expressed with UBE4B and STUB1, UBE4B coprecipitated with STUB1 (Fig. 4d). However, UBE4B did not directly interact with STUB1, as UBE4B did not co-precipitate with STUB1 in the absence of Tau (Fig. 4d). Previous studies demonstrated that STUB1 directly interacts with and ubiquitinates Tau, targeting it for degradation ${ }^{21,27,28}$. UBE4B also directly interacted with Tau protein (Fig. 4e). These results suggested that UBE4B did not directly interact with STUB1, but rather that Tau mediated the interaction between STUB1 and UBE4B.

STUB1 knockdown reduces UBE4B-mediated alleviated $h T a u$ phenotypes in Drosophila. Since the knockdown of STUB1 in neuroblastoma cell lines showed no change in Tau levels in the presence of $U B E 4 B$ (Supplementary Fig. 5b, c), we further examined the importance of STUB1 in the in vivo Drosophila model system. Studies from the in vivo model system showed that the knockdown of STUB1 gene in the eyes of flies expressing $G M R>h T a u+h U B E 4 B$, significantly reduced eye phenotype when compared with $G M R>h T a u+h U B E 4 B$ flies (Fig. 5a, b). Similarly, the knockdown of STUB1 in neurons expressing Elav > $h T a u+h U B E 4 B$ also significantly reduced larval locomotion phenotype when compared with Elav $>$ hTau $+h U B E 4 B$ in neurons (Fig. 5c, d). However, NMJ phenotype showed no change in larvae expressing Elav $>h T a u+h U B E 4 B+S T U B 1-R N A i$ when compared with Elav $>$ hTau + hUBE4B larvae (Fig. 5e, f). These 
a

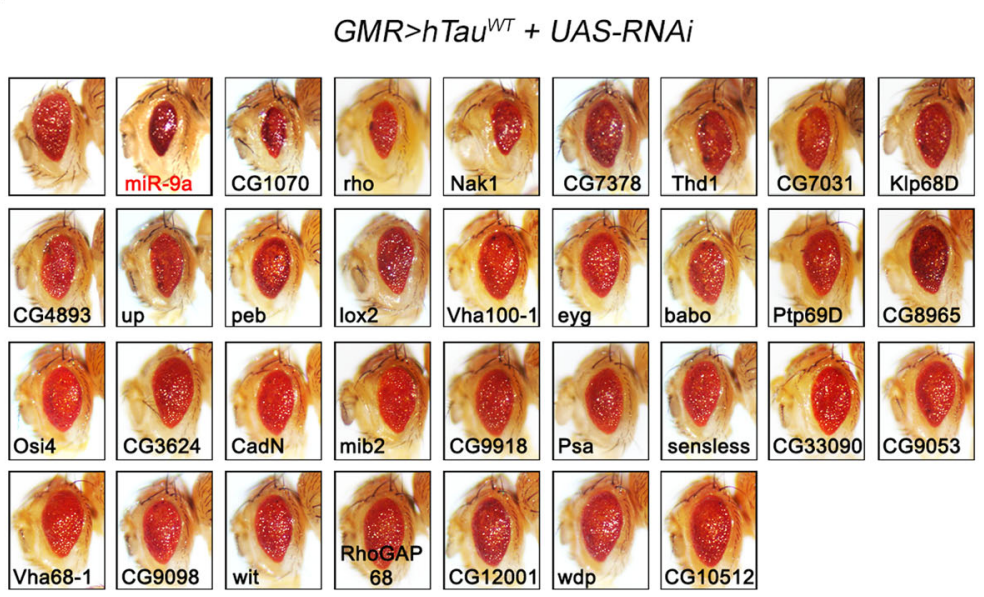

C

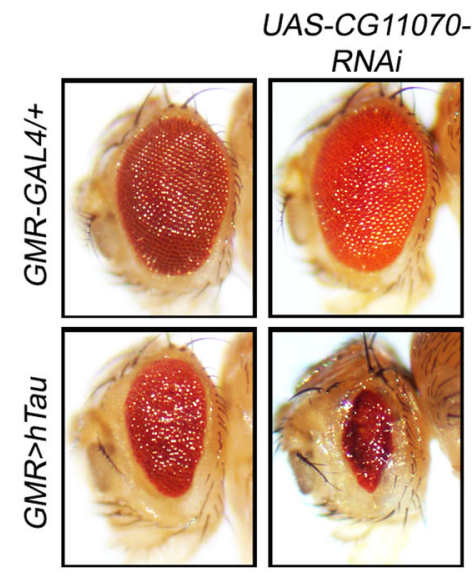

e

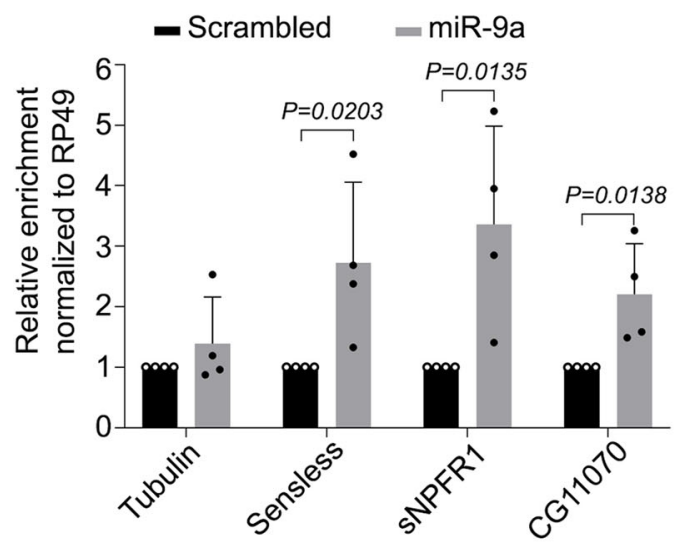

d

f b

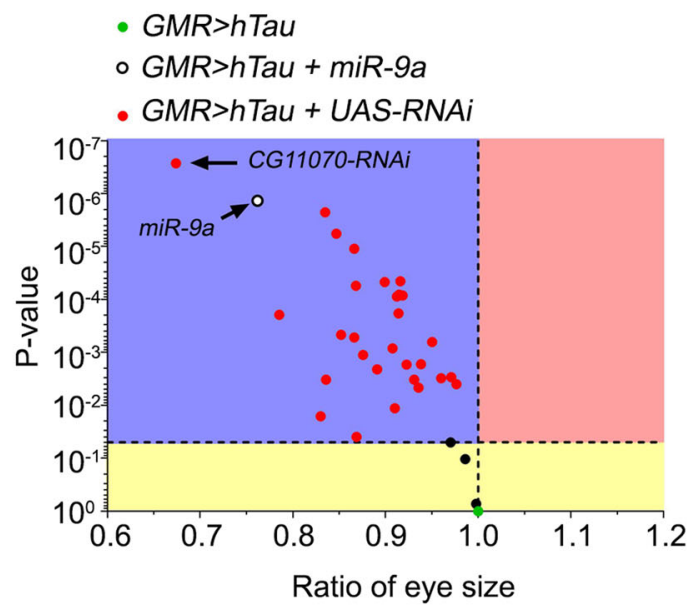

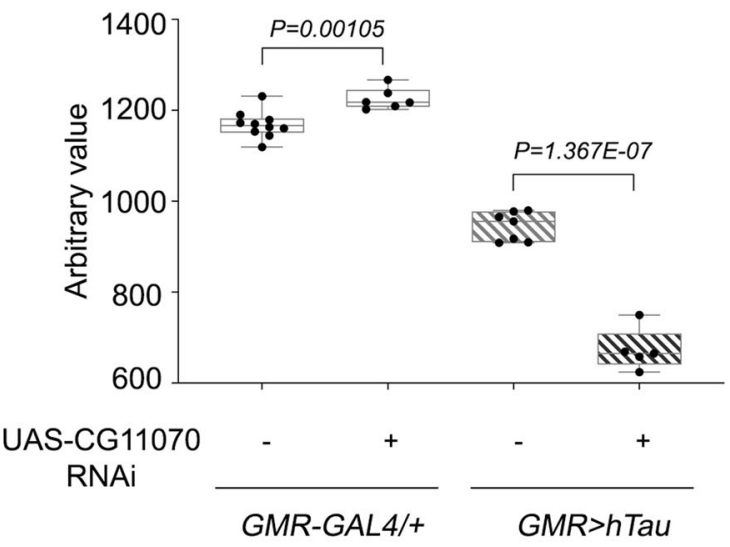

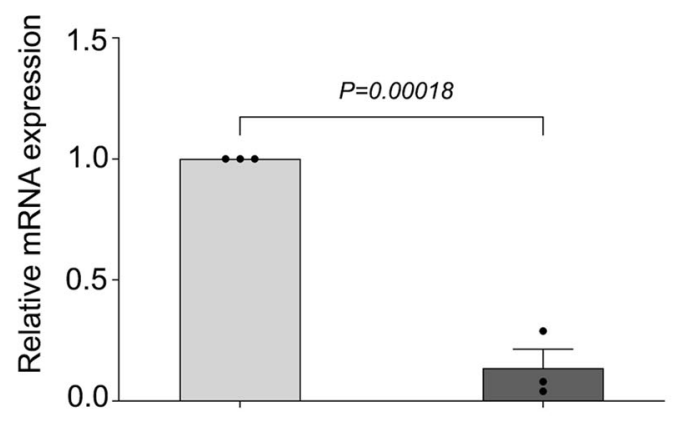

UAS-miR-9a

GMR-GAL4/+

studies indicate that alleviated $h T a u$ phenotypes by $h U B E 4 \mathrm{~B}$ overexpressing are dependent on STUB1 function.

Degradation of Tau by UBE4B and STUB1 in the Tau-BiFC mouse model. To determine whether in vivo overexpression of

$U B E 4 B$ and STUB1 affects Tau degradation, we generated $A A V$ $C M V$-UBE4B and $A A V$-CMV-STUB1 constructs (Fig. 6a). AAVs were delivered to the dentate gyri of Tau-BiFC mice by stereotaxic injection (Fig. 6b, c) and pathological examinations were performed as shown in the work flow (Fig. 6d). In the Tau-BiFC 
Fig. 2 CG11070, a miR-9a target identified from secondary screening, strongly modulated $\boldsymbol{h T a u}$ in Drosophila eyes. a Screening of flies with RNAi knockdown of miR-9a targets in GMR > hTau Drosophila eyes identified a significant reduction in eye size in CG11070-RNAi flies relative to the control. $\mathbf{b}$ Volcano plot of mean eye sizes of flies expressing various miR-9a target gene RNAis in GMR $>h T a$ flies versus their respective $p$-values derived from a one-way analysis of variance followed by pairwise $t$-tests and a Bonferroni correction for multiple comparisons. All points above the dotted line can be considered significant. CG11070-RNAi flies exhibited more severe ocular Tau toxicity, as demonstrated by decreased eye size. $N=3$ biologically independent experiments. c, d The knockdown of CG11070 (CG11070-RNAi) in GMR > hTau flies significantly decreased eye size relative to GMR > hTau controls. $N=5$ biologically independent experiments. In the box plots the whiskers represent the 5 th to 95 th percentile range. Data are presented as the mean \pm s.e.m. Statistical significance was determined with a two-tailed Student's $t$-test. e miRNA-mRNA-RISC pull-down assays in Drosophila S2 cells revealed that miR-9a bound to CG11070 mRNA. Transfection of miR-9a enriched CG11070 mRNA levels, similar to the known miR-9a targets senseless and sNPFR1, as demonstrated by qRT-PCR. $N=4$ biologically independent experiments. Data are presented as the mean \pm s.e.m. Statistical significance was determined with a two-tailed Student's t-test. f Expression of miR-9a using GMR-GAL4 significantly decreased CG11070 expression. $N=3$ biologically independent experiments. Data are presented as the mean \pm s.e.m. Statistical significance was determined with a two-tailed Student's $t$-test. Statistical source data.

mouse model system, Tau oligomerization can be visualized by BiFC fluorescence ${ }^{29}$. Consistent with the in vitro data, the overexpression of either $U B E 4 B$ or STUB1 induced degradation of Tau oligomer, as indicated by decreased Tau fluorescence relative to control (Fig. 6e). Similarly, co-overexpression of $U B E 4 B$ and STUB1 in Tau-BiFC mice further decreased Tau fluorescence (Fig. 6e, f). Similar to reduction of oligomeric Tau levels, phosphorylated Tau levels in the dentate gyrus of Tau-BiFC mice, as detected by the AT8 (p-S202/T205) antibody, were also decreased by the overexpression of either $U B E 4 B$ or $S T U B 1$, and were further decreased by co-expression of $U B E 4 B$ and STUB1 (Fig. 6e and $\mathrm{g}$ ). Additional phosphorylated forms of Tau were detected by AT180 (p-T231) and PHF-1 (p-S396/S404) antibodies. Both pT231 and p-S396/S404 Tau levels were also decreased by the overexpression of either $U B E 4 B$ or STUB1 alone, and were further decreased by co-expression of UBE4B and STUB1 (Supplementary Fig. $6 \mathrm{a}-\mathrm{c})$. These results demonstrated that $U B E 4 B$ and STUB1 additively degraded Tau in vivo.

UBE4B and STUB1-mediated autophagy Tau degradation. The two reported major pathways of Tau degradation are the UPS and $\mathrm{ALS}^{30,31}$. To determine the primary pathway of UBE4B and STUB1-mediated Tau degradation, we co-overexpressed $U B E 4 B$ and STUB1 with Tau in neuroblastoma cells, and treated the cells with either the UPS inhibitor MG132 or the ALS inhibitors. Interestingly, MG132 treatment did not inhibit Tau degradation relative to the control cells (Fig. 7a). In contrast, Chloroquine significantly inhibited Tau degradation (Fig. 7b). In addition, pepstatin A (PEPA) alone and E64D plus PEPA (E64D + PEPA), autophagy inhibitors, significantly inhibited Tau degradation (Fig. 7c), suggesting that ALS was the major pathway of UBE4B and STUB1-mediated Tau degradation in neuroblastoma cells. Our in vitro study showed that the turnover of monomeric Tau molecule by UBE4B/STUB1 is preferentially mediated by autophagy-dependent manner rather than the proteasome pathway in SH-SY5Y cells (Fig. 7a-c).

Because autophagy inhibitor treatment blocked autophagic Tau degradation in vitro, we tested this in the in vivo system by injecting autophagy inhibitors into the dentate gyri of Tau-BiFC mice with co-expression of UBE4B and STUB1 (Fig. 7d, e). We measured oligomeric Tau and phosphorylated Tau levels in the dentate gyrus of Tau-BiFC mice detected with the AT8 antibody (S202/T205) (Fig. 7f-h). Inhibition of ALS by Chloroquine and E64D + PEPA significantly increased oligomeric and phosphorylated Tau (S202/T205) (Fig. 7f-h). Similarly, the phosphorylated forms of Tau p-S396/S404 (PHF-1) and p-T231 (AT180) were also significantly increased in Chloroquine and E64D + PEPAtreated Tau-BiFC mice (Supplementary Fig. 7a-d). Since ALS disruption changes LC3 and p62/SQSTM1 levels, which are inversely correlated with the efficiency of autophagy ${ }^{32}$, we measured LC3 and p62 levels in our model system. Coexpression of $U B E 4 B$ and STUB1 significantly decreased LC3 and p62 levels relative to control in the dentate gyri of Tau-BiFC mice, whereas autophagy inhibitors elevated LC3 and p62 levels relative to the control in the dentate gyri of $U B E 4 B+S T U B 1-$ expressed Tau-BiFC mice (Fig. 7i, j). Furthermore, autophagy inhibitors modulated BECN1 levels compared to the control in the dentate gyri of $U B E 4 B+S T U B 1$-expressed Tau-BiFC mice (Supplementary Fig. 8). Collectively, these results suggested that monomeric and oligomeric Tau degradations by STUB1 and UBE4B are mediated through autophagy pathway rather than the ubiquitin-proteasome system.

\section{Discussion}

In the present study, we demonstrated that Drosophila miR-9a regulated Tau toxicity and increased Tau phosphorylation in the eyes. MiR-9a-mediated Tau toxicity arose through disruption of the $m i R-9 a$ target gene CG11070, the human orthologue of $U B E 4 B$, which when overexpressed mimicked the miR-9a knockdown phenotype. The overexpression of either Drosophila CG11070 or human UBE4B alleviated Tau-associated phenotypes, including increased eye size, improved larval crawling/NMJ phenotypes, and decreased total and phosphorylated Tau levels. Further, we demonstrated that clearance of Tau proteins by UBE4B occurred primarily through ubiquitin-dependent ALS rather than the proteasome system.

Previous studies have demonstrated that miRNAs mediate neurodegeneration, including that of $\mathrm{AD}^{33,34}$. $\mathrm{AD}$-related miRNAs regulate multiple stages of $\mathrm{AD}$ pathology and enhance Tau toxicity 35,36 . Studies in AD cadaver samples demonstrated that $m i R-125 b$ is increased in $\mathrm{AD}$, and the overexpression of $m i R-125 b$ in mice increases Tau hyperphosphorylation by regulating kinases and phosphatases ${ }^{37}$. Also, downregulation of $m i R-132 / 212$ promotes Tau phosphorylation, which in turn enhances $\mathrm{AD}$ phenotypes $^{38}$. In addition to these miRNAs, $m i R-138$ regulates Tau phosphorylation through GSK3 $\beta^{39}$ and miR-922 affects Tau phosphorylation through UCHL1 ${ }^{40}$. Functional analysis of $m i R-9$ has revealed its involvement in the regulation of neuronal progenitor cells and differentiation of neuronal cells during development ${ }^{41}$. In AD patients, $m i R-9$ expression is elevated ${ }^{42}$. In Drosophila, $m i R-9 a$ plays an important role in the development of sensory organs by suppressing its target gene senseless ${ }^{24}$ and also regulates body growth through $s N P F R$ signaling ${ }^{23}$. In the Drosophila Tau AD model, we observed increased Tau toxicity when $m i R-9 a$ was overexpressed. The Drosophila miR-9a binding sequence was $100 \%$ identical to that of mammalian miR-9 (Fig.1e). Our studies clearly demonstrated that $m i R-9 a$ regulated Tau toxicity, exacerbating the rough eye phenotype and significantly decreasing eye size. 
a

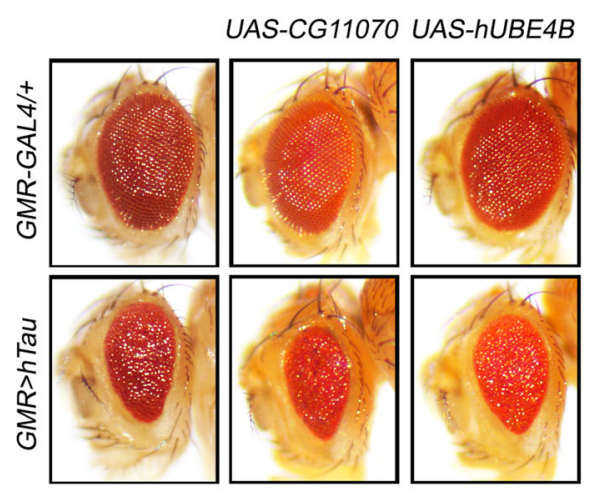

C

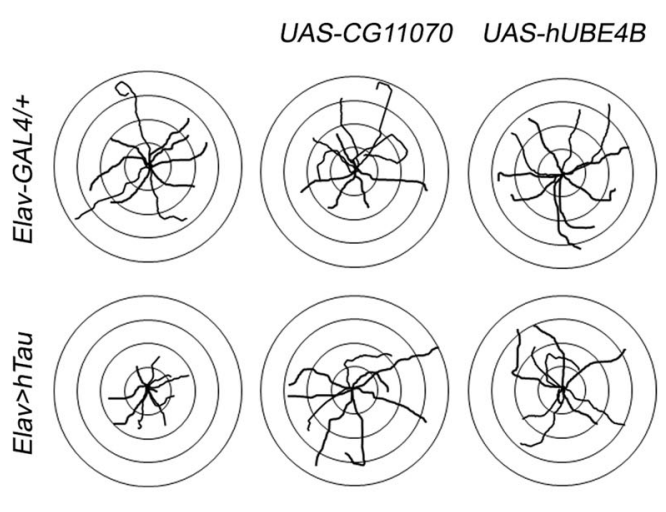

e

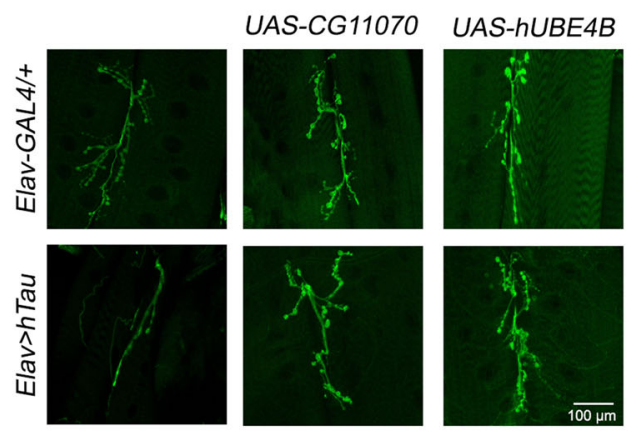

b

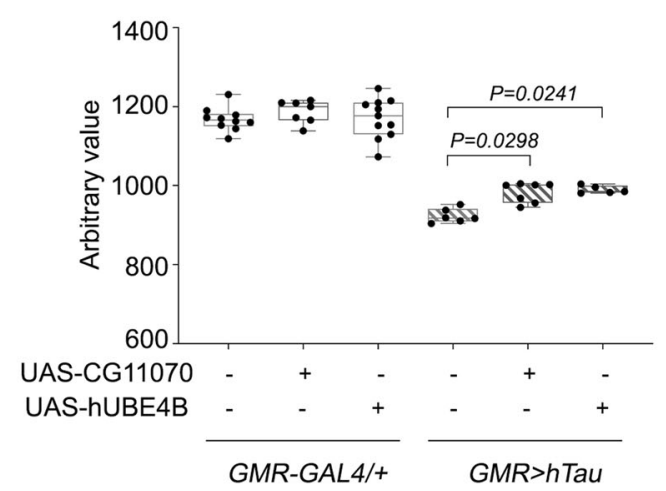

d

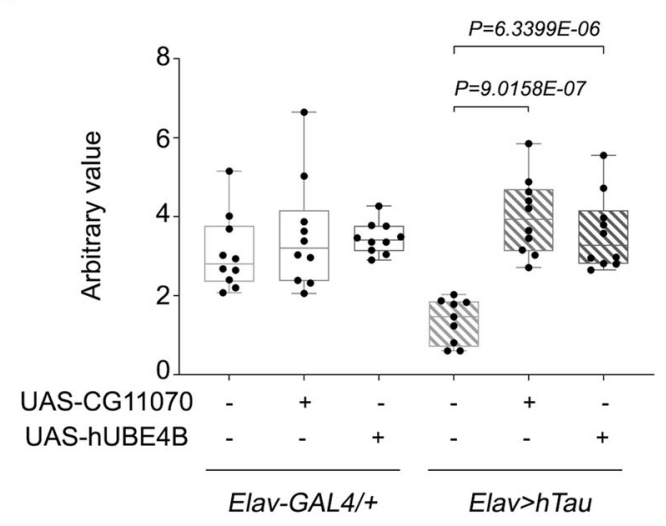

f
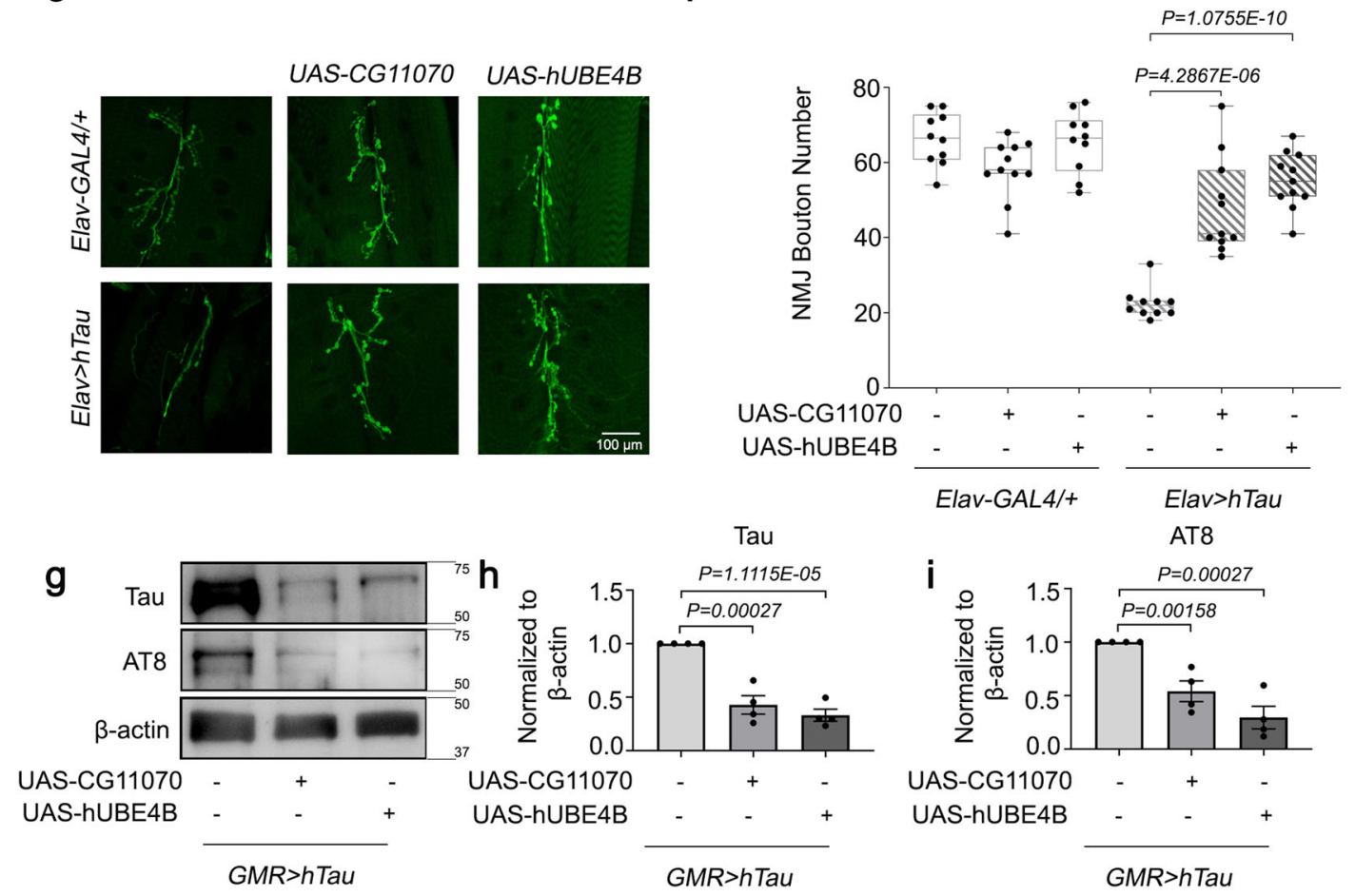

$m i R-9 a$ targets a multitude of genes that are involved in diverse cellular processes, and we used in silico analysis to identify 34 putative targets, which were predicted as miR-9a target genes using three separate in silico platforms. We then performed a secondary screening of the putative $34 \mathrm{miR}-9 a$ target genes to identify a distinct gene, CG11070, as a strong modifier of $h$ Tau (Fig. 2). Mammalian orthologues of Drosophila CG11070 include UBE4A and UBE4B. UBE4B, but not UBE4A, contained miR-9 binding sequences in its $3^{\prime}$ UTR like those of CG11070 (Supplementary Fig. 3b), and is extensively expressed in the brain ${ }^{43}$. We 
Fig. 3 The overexpression of Drosophila CG11070 and its mammalian orthologue UBE4B alleviated hTau phenotypes in Drosophila. a, b Eye-specific overexpression of Drosophila CG11070 or its mammalian orthologue UBE4B in GMR $>h T a u$ flies increased eye size relative to GMR $>h T a u$ controls. $N=5$ biologically independent experiments. In the box plots the whiskers represent the 5th to 95th percentile range. c, d Neuronal overexpression of Drosophila CG11070 or its mammalian orthologue UBE4B using Elav-Gal4 in Elav > hTau flies significantly increased larval crawling. $\mathrm{N}=10$ biologically independent experiments. In the box plots the whiskers represent the 5th to 95th percentile range. e, $\mathbf{f}$ Neuronal overexpression of Drosophila CG11070 or its mammalian orthologue UBE4B using Elav-Gal4 in Elav > hTau flies significantly increased synaptic bouton numbers relative to Elav >hTau controls. Scale bar $100 \mu$ m. $N=10$ biologically independent experiments. In the box plots the whiskers represent the 5 th to 95th percentile range. g-i Western blotting revealed that ocular overexpression of Drosophila CG11070 or its mammalian orthologue UBE4B in GMR > hTau flies significantly reduced total and phosphorylated Tau protein levels relative to $G M R>h T a u$ controls. $N=4$ biologically independent experiments. Data are presented as the mean \pm s.e.m. Statistical significance was determined with a two-tailed Student's $t$-test. Statistical source data.

therefore considered $U B E 4 B$ as the mammalian orthologue of Drosophila CG11070 in the context of $m i R-9 a$ regulation of Tau toxicity. The overexpression of UBE4B or CG11070 in Drosophila not only alleviated $h$ Tau eye neurodegenerative phenotypes, larval crawling defects, and synaptic dysfunction in NMJs (Fig. 3a, f), but also decreased total and phosphorylated Tau levels (Fig. 3g-i, Supplementary Fig. $3 \mathrm{c}-\mathrm{e}$ ). These results indicated that Tau degradation was strongly regulated by UBE4B/CG11070. Since Tau is involved in maintaining the stability of microtubules in neurons, the degradation of Tau proteins affects microtubule stability as seen in tauopathy and Alzheimer's disease models. However, recent studies have shown that reduction in Tau proteins leads to increased accumulation of MAP6 proteins on the microtubules and enhances the stability of microtubules in neurons ${ }^{44}$. The rescue phenotype seen in UBE4B/CG1170 may be a result of compromised function of Tau and MAP6 proteins in the neurons. Reduction in Tau proteins leads to reduced pTau levels in UBE4B/CG11070 rescue animals, due to increased ubiquitination of Tau proteins. Studies have shown that the acetylation of Tau inhibits degradation of phosphorylated Tau and contributes to increased tauopathy ${ }^{45}$. Both ubiquitination and acetylation shares common Lys residue Lys $280^{46}$ which is involved in stability of Tau proteins. The UBE4B proteins are distinct from E3 ligases in their U-Box domains, which possess both E3 ligase and E4 ligase activities ${ }^{47}$. UBE4B ligase ubiquitinates the tumor suppressor genes p53, p63 and p73, and Wallerian degeneration proteins in conjunction with various E3 ligases $^{48}$. In the Drosophila spinocerebellar ataxia type 3 model, UBE4B ubiquitinates ataxin, targeting it for proteasomal degradation. However, the dominant-negative UBE4B mutation enhances neurodegeneration ${ }^{49}$. These previous studies suggest that UBE4B potentiates the degradation of various proteins through the UPS, unlike our current findings, which suggest synergistic regulation of Tau degradation through the ALS in conjunction with STUB1.

Prior reports have demonstrated that Tau is ubiquitinated by two E3 ubiquitin ligases, STUB1 $1^{21,27,28}$ and TRAF6 ${ }^{50}$, both of which are colocalized with NFTs in AD brains. In the present study, we found that Tau was ubiquitinated by the E3 ligase STUB1 in the presence of $U B E 4 B$, a further ubiquitin conjugation factor (Fig. 4a). In addition, Tau degradation was increased by $U B E 4 B$ expression, and further increased by STUB1 and UBE4B co-expression (Fig. 4b, c). Studies on Tau degradation have shown that the proteasomal pathway plays a crucial role. Inhibition of proteasome in HEK cells increases the levels of fulllength Tau proteins ${ }^{50}$. Similar results were seen in SH-SY5Y cells with increased accumulation of both full-length Tau protein and mutant P301L Tau protein levels when proteasome degradation is inhibited ${ }^{51,52}$. However, from our studies, STUB1- and UBE4Bmediated Tau degradation was inhibited by the autophagy inhibitors such as Chloroquine, PEPA, and E64D (Fig. 7b, c) but not by the proteasomal inhibitor MG132 (Fig. 7a), suggesting that STUB1/UBE4B-mediated Tau degradation was facilitated by autophagy rather than the UPS. In FTDP-17 mutant P301S mice, trehalose treatment promotes autophagy with reduced insoluble and pTau proteins ${ }^{53}$. Similarly, the knockout of Atg7 (autophagy marker) in neurons give rise to increased pTau levels and neurodegeneration with aging ${ }^{54}$. A number of autophagy inhibitors, including chloroquine, $\mathrm{NH}_{4} \mathrm{Cl}, 3$-methyl adenine (3-MA), and cathepsin inhibitors, delay Tau degradation and enhance the formation of high molecular weight Tau aggregates ${ }^{55,56}$. Contrastingly, the autophagy inducer rapamycin facilitates insoluble Tau degradation, alleviating Tau toxicity in Drosophila ${ }^{30}$. Trehalose, an mTOR-independent autophagy activator ${ }^{31}$, improves neuronal survival by decreasing Tau aggregation in tauopathy mouse model ${ }^{57,58}$. This suggests that basal autophagy activity is essential for prevention of neuronal Tau aggregate accumulation, which is regulated in part by UBE4B activation. Considering autophagy inhibitors not only modulate neuronal autophagy pathway but also affect neuroinflammation pathway, it remains to be determined whether other neuroinflammatory pathways are involved in Tau pathology beyond the autophagy pathway in future studies.

Lys63-linked polyubiquitination of Tau facilitates the formation of disease-associated Tau inclusions, which are preferentially cleared by the ALS ${ }^{59}$, while Lys48-linked polyubiquitinated Tau is likely degraded by the UPS ${ }^{60}$. Lys63 ubiquitinated substrates are recognized by autophagy receptors present on autophagosomes, mediating ALS-dependent degradation ${ }^{61-63}$. Recent studies have shown that the knockdown of UBE4B affects Lys48 and Lys63 polyubiquitination in Tax binding proteins and Tax mediated activation of NF- $\mathrm{KB}^{64}$. Therefore, in the context of the present study, UBE4B acted synergistically with STUB1 to facilitate Tau degradation by ALS, and could polyubiquitinate Tau proteins by Lys63 ubiquitin linkage. The specific ubiquitin chain linkage for Tau ubiquitination by STUB1/UBE4B is a potential target for lysosomal targeting of Tau. However, the mechanism underlying ALS-mediated Tau degradation remains to be elucidated in future studies.

Tau oligomers play a central role in tauopathies. To understand the importance of Tau oligomers in vivo, Bimolecular Fluorescence Complementation (BiFC) technology was applied to visualize Tau oligomerization ${ }^{65}$. In this system, full-length human Tau protein is fused to the non-fluorescent $\mathrm{N}$ - and C-terminal termini of Venus fluorescent protein. In the transgenic TauP301L-BiFC mouse model, Venus fluorescence is activated only when Tau is aggregated ${ }^{29}$. In our studies using the TauP301L-BiFC mouse model, the overexpression of UBE4B, $S T U B 1$, or both significantly reduced the fluorescence generated by Tau oligomers (Fig. 6). Similarly, Tau phosphorylation was also decreased in the dentate gyri of TauP301L-BiFC mice with the overexpression of $U B E 4 B, S T U B 1$, or both. These results indicate that Tau oligomers, a primary cause of tauopathy, can be degraded by UBE4B and STUB1 in vivo. In TauP301L-BiFC mice, treatment with the autophagy inhibitors (Chloroquine, PEPA, and E64D) increased oligomeric and phosphorylated Tau levels 


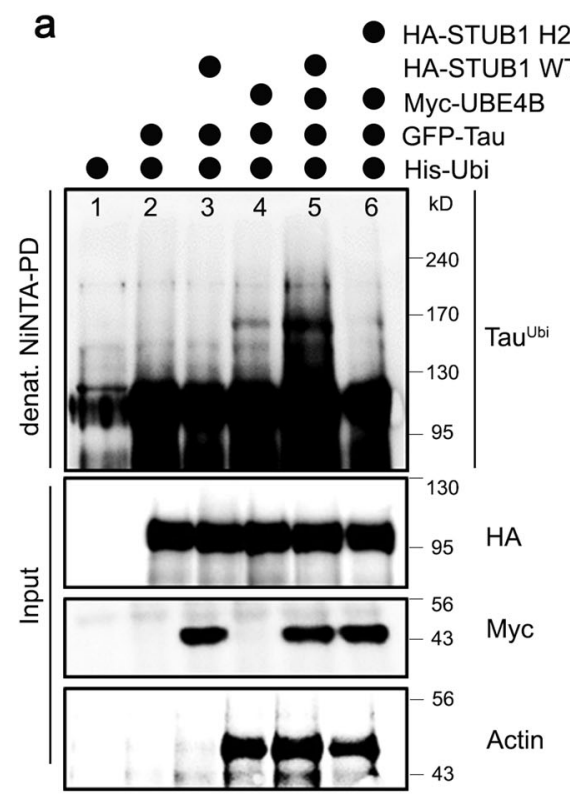

b
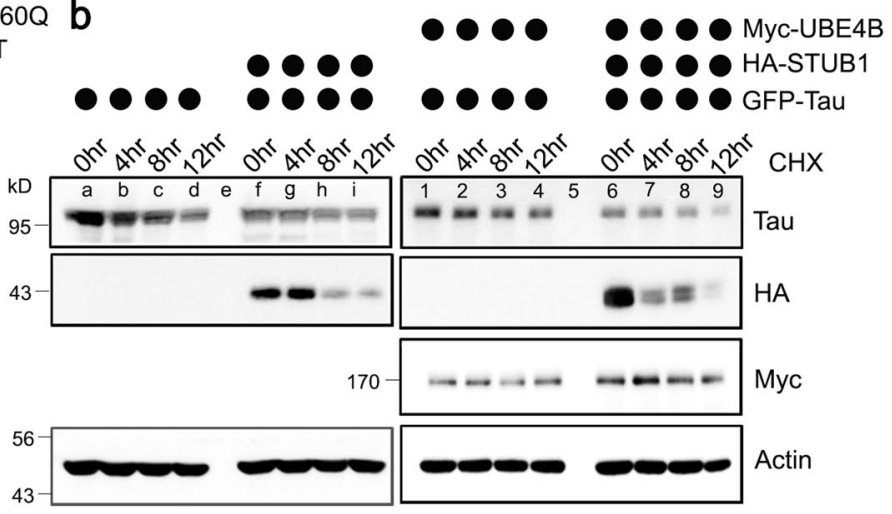

C

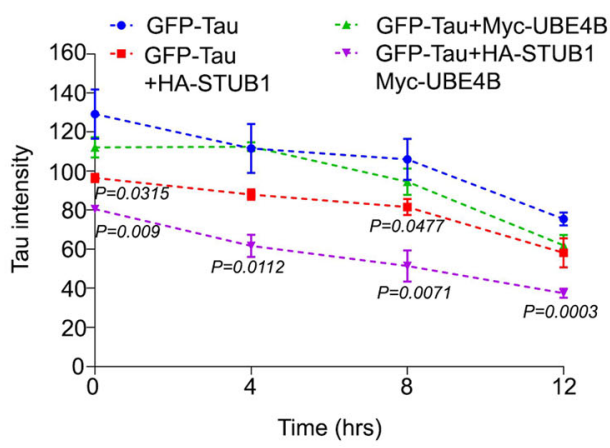

d

e
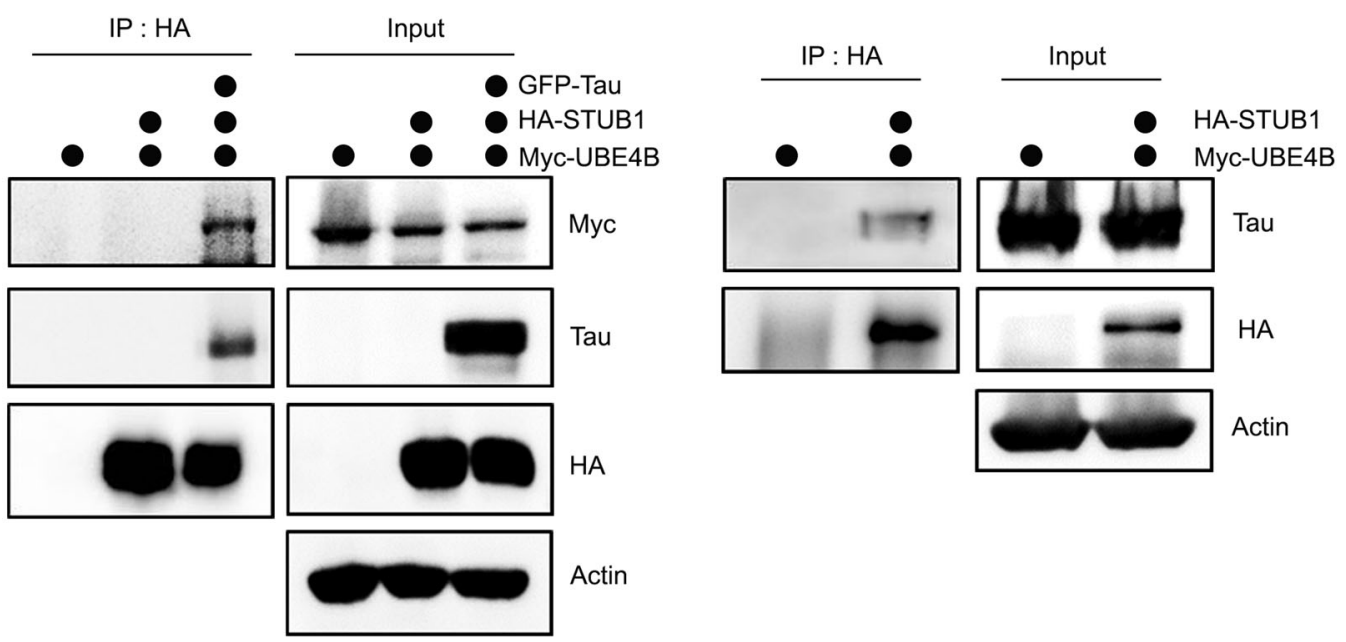

Fig. 4 Tau was ubiquitinated and degraded by UBE4B and STUB1 in mammalian neuroblastoma cells. a Tau was co-expressed with His-Ubiquitin, UBE4B, and STUB1 WT or dominant-negative mutant STUB1 ${ }^{260 Q}$ in SH-SY5Y neuroblastoma cells. Ubiquitinated Tau was significantly increased by co-expression of UBE4B and STUB1 (lane 5), but not by expression of UBE4B (lane 4) or STUB1 (lane 3) alone. Tau ubiquitination by co-expression of UBE4B and STUB1 required the ligase activity of STUB1 (lanes 5 and 6). b, c Tau protein degradation was enhanced by co-expression of UBE4B and STUB1 (lane 6-9) compared with expression of UBE4B (lane 1-4) or STUB1 (lane $\mathrm{f}-\mathrm{i}$ ) alone. Quantification of Tau levels was normalized to the amount of $\beta$-actin protein in each case. Data represent the mean \pm s.e.m of three independent experiments $\left({ }^{\star} P<0.05,{ }^{\star \star} P<0.005,{ }^{\star \star \star} P<0.001\right.$ two-tailed Student's $t$-test). $\mathbf{d} \cup B E 4 B$ was co-expressed with HA-STUB1 and Tau in SH-SY5Y cells and immunoprecipitated on anti-HA-agarose beads. UBE4B did not directly interact with STUB1 in the absence of Tau, but indirectly interacted with STUB1 in the presence of Tau. e HA-UBE4B was co-expressed with Tau in SH-SY5Y cells and immunoprecipitated on anti-HA-agarose beads. Co-precipitated Tau was detected by Western blot, revealing that Tau directly interacted with UBE4B. All western blots were performed more than three times. Statistical source data.

(Fig. 7b, c, f-h, Supplementary Fig. 7). Notably, while the overexpression of UBE4B and STUB1 decreased protein levels of LC3, p62, and BECN1, autophagy markers, autophagy inhibitors significantly elevated LC3, p62, and BECN1 levels in the dentate gyri of AAV-STUB1/UBE4B injected TauP301L-BiFC mice (Fig. 7i-1 and Supplementary Fig. 8). Collectively, these results further suggested that UBE4B promoted oligomeric Tau clearance via ALS through a STUB1-dependent mechanism. 
a

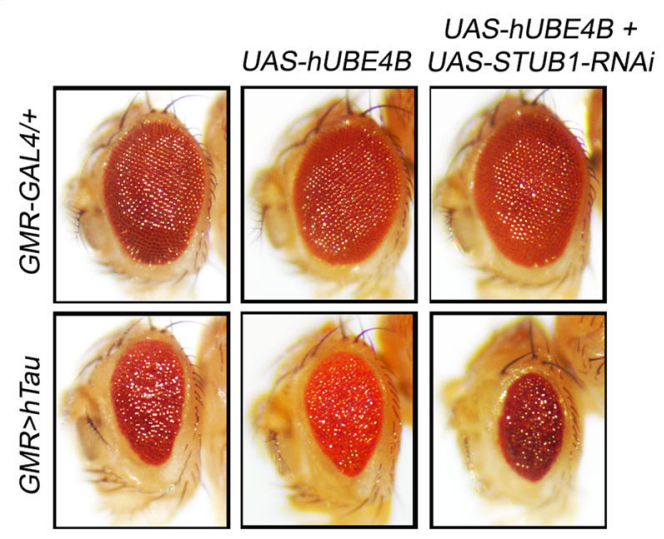

C

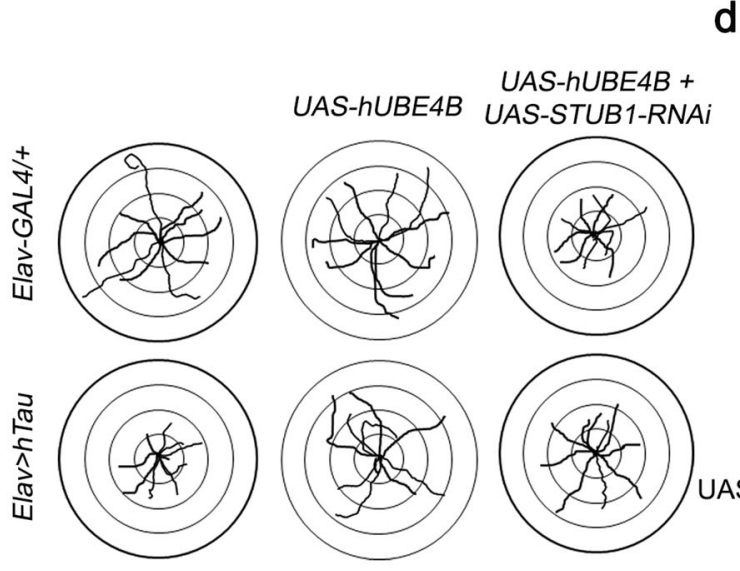

e

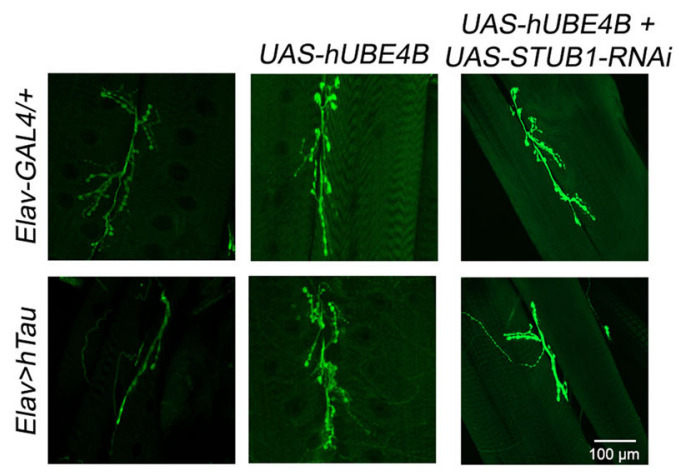

b

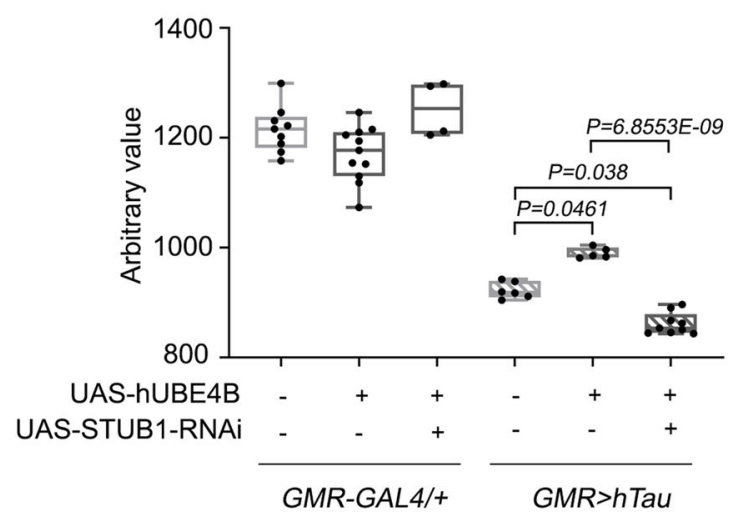

d

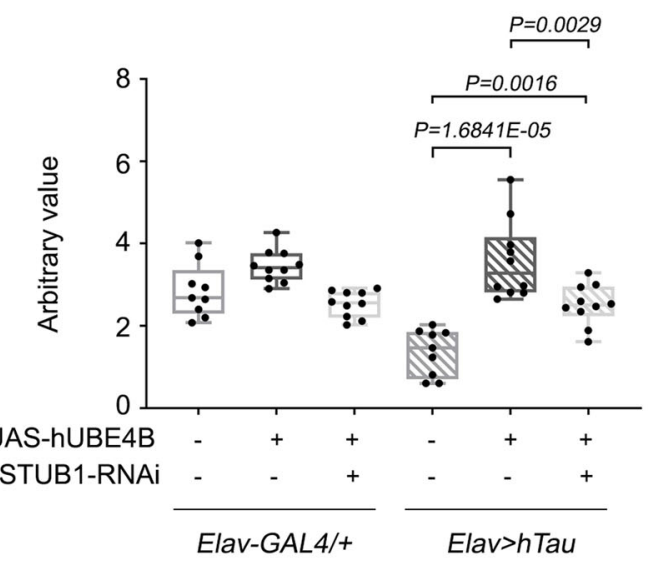

f

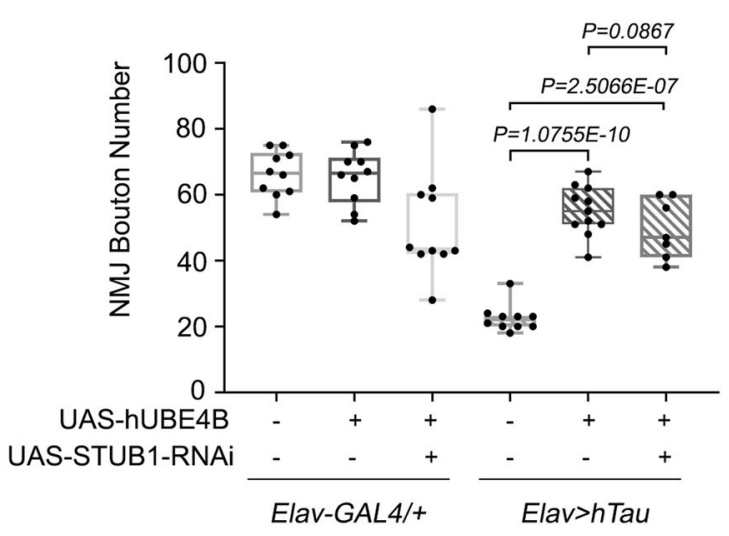

Fig. 5 STUB1 knockdown reduces UBE4B-mediated alleviated hTau phenotypes in Drosophila. a, b Eye-specific knockdown of Drosophila STUB1 significantly reduced eye size relative to $G M R>h T a u+h U B E 4 B$ flies. $N=5$ biologically independent experiments. In the box plots the whiskers represent the 5th to 95th percentile range. c, d Neuronal knockdown of Drosophila STUB1 using Elav-Gal4 in Elav > hTau $+h$ UBE4B flies significantly reduced larval crawling. $N=10$ biologically independent experiments. In the box plots the whiskers represent the 5th to 95th percentile range. e, $\mathbf{f}$ Neuronal knock down of Drosophila STUB1 using Elav-Gal4 in Elav > hTau $+h$ UBE4B flies significantly reduced synaptic bouton numbers relative to Elav $>h T a u$ controls. Scale bar $100 \mu \mathrm{m} . N=10$ biologically independent experiments. In the box plots the whiskers represent the 5th to 95th percentile range. Data are presented as the mean \pm s.e.m. Statistical significance was determined with two-tailed Student's $t$-test. Statistical source data.

In the present study, we identified that Drosophila CG11070 and its mammalian orthologue $U B E 4 B$, which are targets of $m i R-9 a / m i R-9$, rescued human Tau phenotypes in flies by decreasing total and phosphorylated Tau levels. In mammalian neuroblastoma cells, UBE4B-mediated Tau clearance was accelerated by co-expression of STUB1, which encodes an ubiquitin E3 ligase for Tau. In the dentate gyri of Tau BiFC mice, the overexpression of UBE4B and STUB1 also decreased oligomeric Tau and phosphorylated Tau levels. These Tau degradations occurred primarily via ALS in mammalian in vitro and in vivo systems. These results demonstrated that UBE4B promoted autophagy-mediated Tau degradation synergistically with STUB1, providing an innovative therapeutic approach for AD. 
a

b

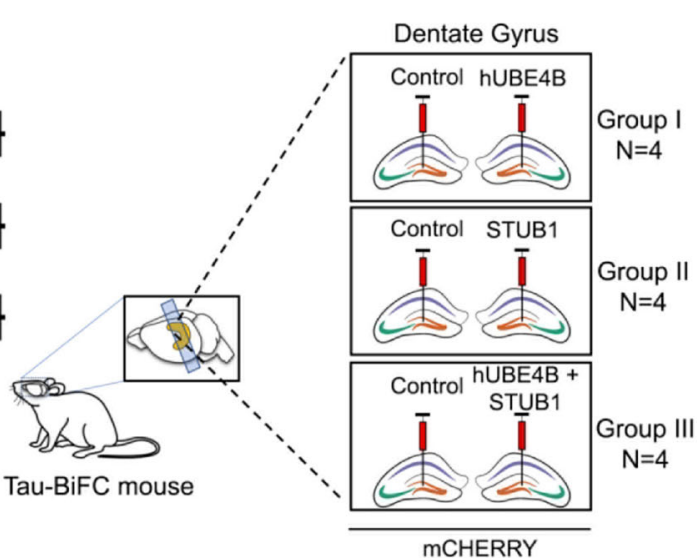

C

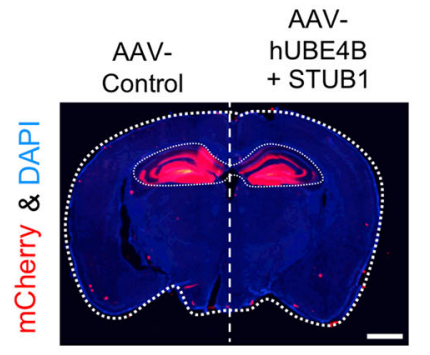

e

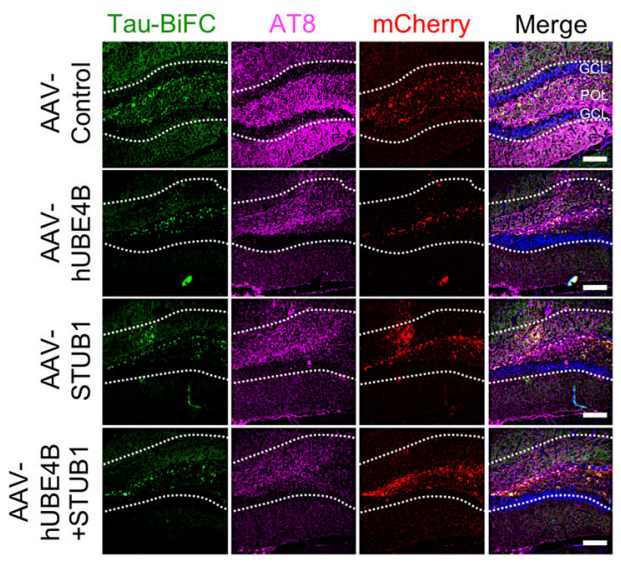

d

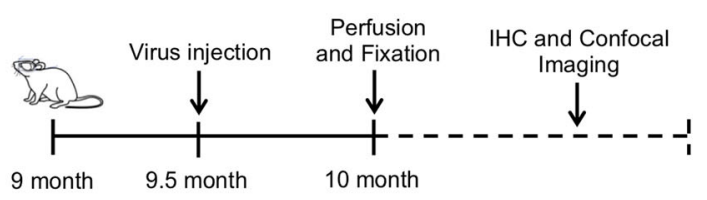

f

g

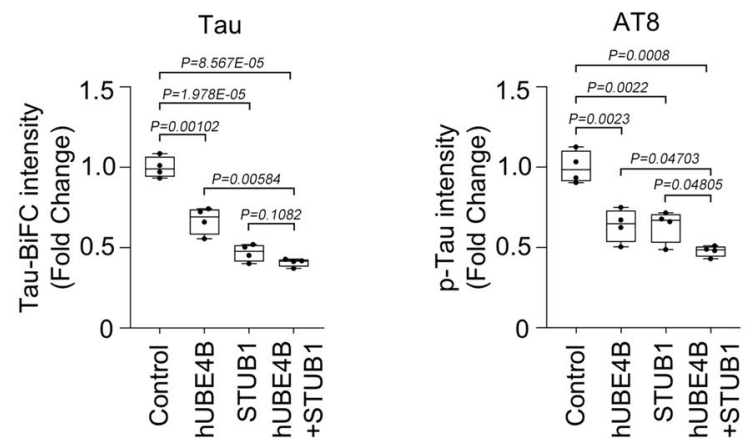

Fig. 6 Tau oligomers were degraded by UBE4B and STUB1 in the Tau-BiFC mouse model. a Schematic representation of $A A V-C M V$ - $m C h e r r y$ and $A A V$ $U B E 4 B-m C h e r r y$ virus constructs. b Schematic illustration of $A A V$-CMV-mCherry or $A A V$-UBE4B-mCherry virus delivery into the dentate gyrus of Tau-BiFC mice. c $A$ fluorescence staining image indicating the foci (red) of $A A V-C M V$ - $m$ Cherry or $A A V-U B E 4 B+S T U B 1$ - $m C h e r r y$ virus delivery in the dentate gyrus and hippocampus of Tau-BiFC mice. Scale bar (white), $1 \mathrm{~mm}$. d Schematic illustration of the work flow for virus injection and pathological examination in Tau-BiFC mice. e AAV-UBE4B and AAV-STUB1 decreased oligomer Tau-BiFC and pTau (S202/T205) levels in the dentate gyrus compared with the control. $\mathrm{GCL}$, granular cell layer; $\mathrm{POL}$, polymorphic layer. Scale bars (white), $80 \mu \mathrm{m}$. These experiments were performed four times. $\mathbf{f}, \mathbf{g}$ Densitometry analysis revealed that $A A V-U B E 4 B$ and STUB1 significantly decreased both Tau-BiFC and pTau (S202/T205) levels in the dentate gyrus relative to the control ( $A A V$-Cont $N=4 ; A A V-U B E 4 B N=4 ; A A V-S T U B 1 N=4 ; A A V-U B E 4 B+A A V-S T U B 1, N=4 ; N=4$ biologically independent animals), respectively. In the box plots the whiskers represent the 5 th to 95 th percentile range. Data are presented as means as \pm s.e.m. Statistical significance was determined with two-tailed Student's $t$-test. Statistical source data.

\section{Methods}

Drosophila culture and stocks. Drosophila melanogaster were maintained at $25^{\circ} \mathrm{C}$ on standard cornmeal, yeast, sugar, and agar medium. UAS-hTau, GMR-GAL4, and ElavGAL4 fly lines were obtained from Bloomington Stock Centre (Bloomington, USA). miR-9a target RNAi stocks were obtained from Bloomington Stock Centre (Bloomington, USA) and Vienna Drosophila Research Centre (Vienna, Austria). pUAS-CG11070 and $p U A S-U B E 4 B$ flies were generated by the p-element-mediated germline transformation method with cDNA containing the coding regions of CG11070 or UBE4B.

Cell culture and transfections. SH-SY5y neuroblastoma cells were maintained in Dulbecco's modified Eagle's medium (DMEM) supplemented with 10\% heatinactivated fetal bovine serum, penicillin $(10 \mathrm{U} / \mathrm{mL})$ and streptomycin $(100 \mu \mathrm{g} / \mathrm{mL})$. Cells were incubated at $37^{\circ} \mathrm{C}$ in $5 \% \mathrm{CO}_{2}$ and transfected with desired plasmids using Effectene transfection reagent (Qiagen) following the manufacturer's instructions. SiRNAs were transfected with lipofectamine 2000 transfection reagent.

Mouse model and virus injection. Male TauP301L-BiFC mice were a kind gift of Dr. Yunkyung Kim (KIST, KOR $)^{29}$. Brain specimens of TauP301L-BiFC mice were prepared as previously described ${ }^{66}$. AAV-CMV-UBE4B and $A A V-C M V-S T U B 1$ viruses were injected using a stereotaxic micro-injector (Stoelting Co.). Control groups were injected with $A A V-C M V$. Neuropathological experiments were performed at 2 weeks after injection. Mice were housed on a 12:12 h light-dark cycle and maintained at $18-23{ }^{\circ} \mathrm{C}$ with humidity between 40 and $60 \%$ in pathogen-free facilities at Korea Institute of Science and Technology. All animal experiments were performed in accordance with the National Institutes of Health Guide for the Care and Use of Laboratory Animals of the Korea Institute of Science and Technology. 


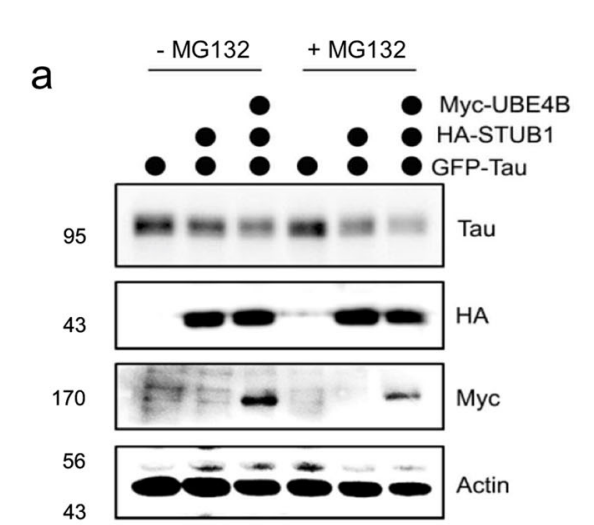

d

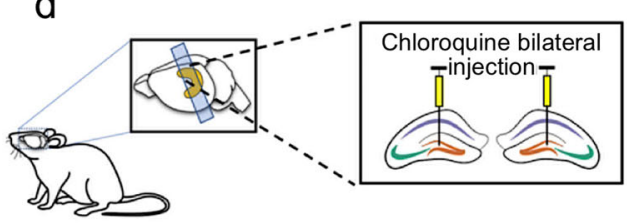

$f$

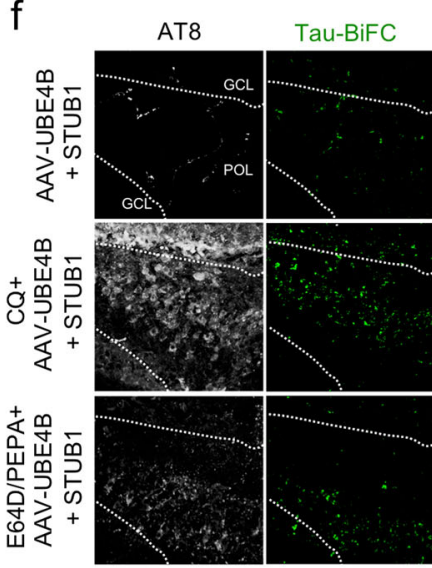

i

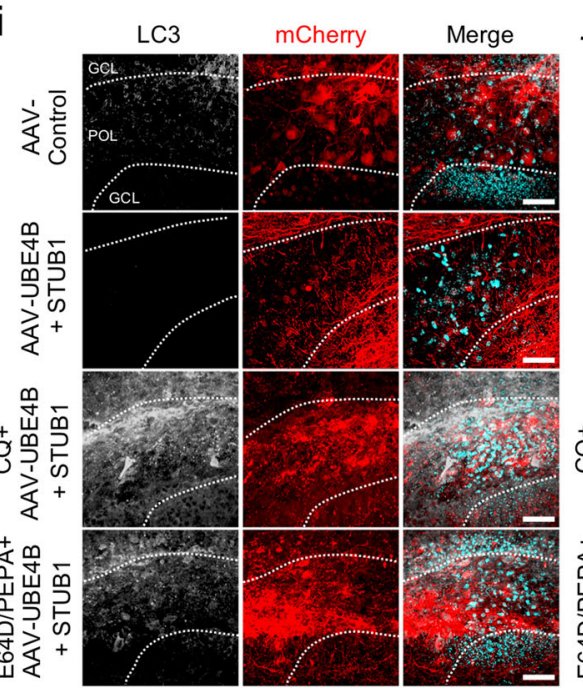

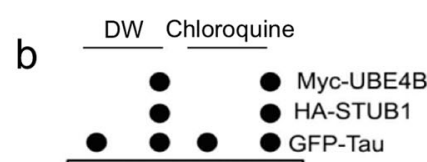
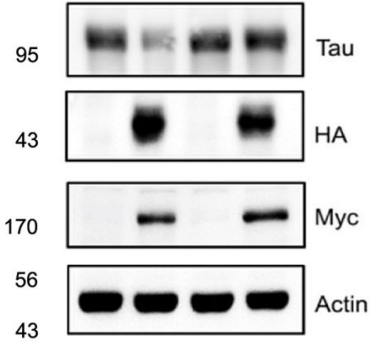

e

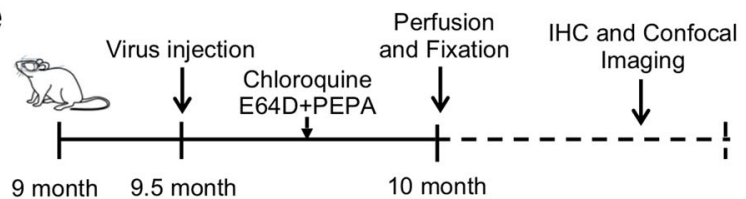

g
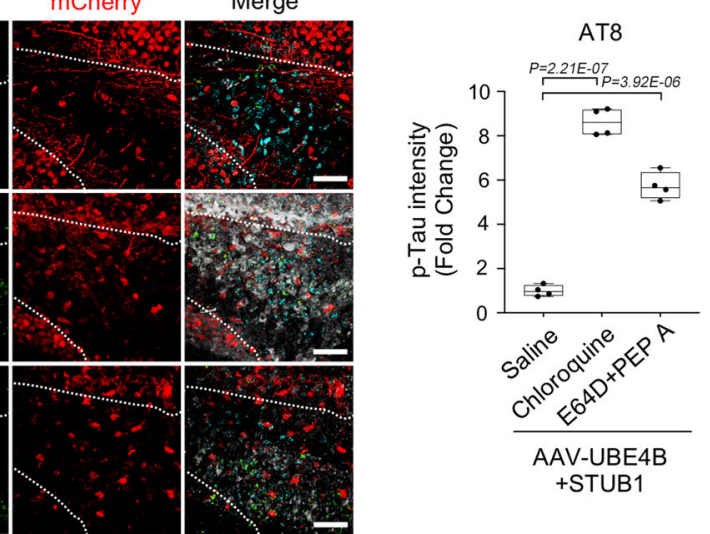

h

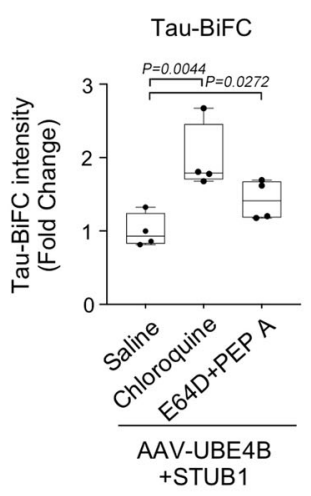

k
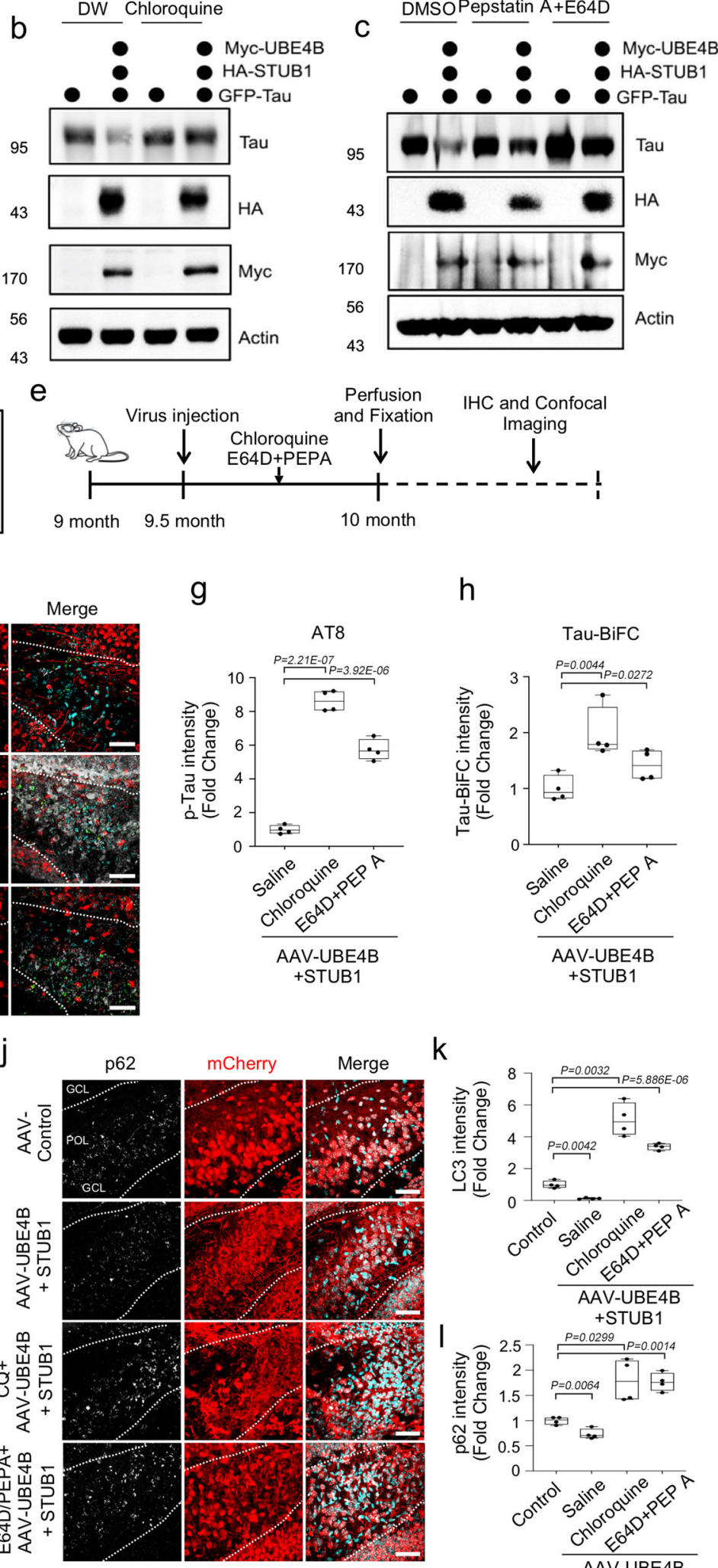

Pepstatin A 
Fig. 7 Tau was degraded by UBE4B and STUB1 primarily via autophagy. a Treatment of SH-SY5Y cells with the proteasome inhibitor MG132 did not affect Tau degradation mediated by UBE4B/STUB1. b Treatment with chloroquine (CQ), an autophagy inhibitor, affected Tau degradation by UBE4B/STUB1. c Pepstatin A (PEPA) and E64D, autophagy inhibitors, inhibited Tau degradation by UBE4B/STUB1. All western blots were performed three times. $\mathbf{d}$ Schematic illustration of autophagy inhibitor injection to the dentate gyrus of Tau-BiFC mice. e Schematic illustration of the work flow for autophagy inhibitor injection and pathological examination in Tau-BiFC mice. $\mathbf{f}$ CQ and E64D plus PEPA (E64D + PEPA) increased pTau (S202/T205) levels in the dentate gyrus relative to saline-injected controls. GCL granular cell layer, POL polymorphic layer. $\mathbf{g}$, $\mathbf{h}$ Densitometry analysis revealed that chloroquine and ED64 + PEPA significantly increased both pTau (S202/T205) and Tau-BiFC levels in the dentate gyrus relative to saline-injected controls ( $A A V-U B E 4 B+A A V-S T U B 1$ (saline control), N = 4; $C Q+A A V-U B E 4 B+A A V-S T U B 1, N=4 ; E 64 D / P E P E A+A A V-U B E 4 B+A A V-S T U B 1, N=4 ; N=4$ biologically independent animals). In the box plots the whiskers represent the 5th to 95th percentile range. $\mathbf{i} A A V-U B E 4 B$ and AAV-STUB1 decreased LC3 levels in the dentate gyrus relative to control. Scale bars (white): $40 \mu \mathrm{m}$. j $A A V$-UBE4B and AAV-STUB1 decreased p62 levels in the dentate gyrus relative to control. Scale bars (white): $40 \mu \mathrm{m}$. $\mathbf{k}$ Densitometry analysis revealed that autophagy inhibitors significantly increased LC3 levels in the dentate gyrus relative to control ( $A A V-C o n t r o l, N=4 ; A A V-U B E 4 B+A A V-S T U B 1, N$ $=4 ; C Q+A A V-U B E 4 B+A A V-S T U B 1, N=4 ; E 64 D / P E P A+A A V-U B E 4 B+A A V-S T U B 1, N=4 ; N=4$ biologically independent animals). In the box plots the whiskers represent the 5th to 95th percentile range. I Densitometry analysis revealed that autophagy inhibitors significantly increased p62 levels in the dentate gyrus relative to control ( $A A V$-Control, $N=4 ; A A V-U B E 4 B+A A V-S T U B 1, N=4 ; C Q+A A V-U B E 4 B+A A V-S T U B 1, N=4 ; E 64 D / P E P A+A A V-U B E 4 B+A A V-$ $S T U B 1, N=4 ; N=4$ biologically independent animals). In the box plots the whiskers represent the 5 th to 95 th percentile range. Data are presented as means \pm s.e.m. Statistical significance was determined with a two-tailed Student's $t$-test. Statistical source data. Each exact $p$ value was listed in Statistical source data.

paper and placed on a $2 \%$ agar grape juice-coated petri dish. Each genotype was allowed to crawl freely for $90 \mathrm{~s}$. To quantify crawling distance, lines were drawn to track the crawling larvae, and total distance was measured using Image J v1.44 software. Approximately 10-20 animals were tested for each genotype ${ }^{25,26}$.

Immunohistochemistry of neuromuscular junction. Third instar larvae were dissected in PBS, fixed in $4 \%$ formaldehyde in PBS for $15 \mathrm{~min}$, and washed three times in $0.1 \%$ Triton X-100 in PBS. FITC-conjugated anti-HRP was used at 1:100 and incubated for $1.5 \mathrm{~h}$ at room temperature. Larvae were mounted in Slow Fade Antifade media. Confocal images were captured using Zeiss confocal microscopes. Quantification of the NMJ was performed by counting the number of boutons in each genotype using Image J v1.44 software with cell counter plugin ${ }^{25,26}$.

Quantitative PCR. The heads of 20 adult Drosophila per group were collected and total RNA was isolated with Trizol reagent. After treating the RNA samples with RNase-free DNase I, cDNA was synthesized using the SuperScript III First-Strand Synthesis System (TAKARA, Japan). Quantitative reverse transcription-PCR (qRT-PCR) analysis was performed using a StepOnePlus Sequence Detection System (BioRAD, USA) with SYBR Green PCR Core reagents (BioRAD). Each experiment was performed at least in triplicate. The comparative cycle threshold was utilized to quantify the fold change of each specific mRNA after normalizing to $r p 49$ levels.

miRNA-mRNA pull-down assay. The miRNA-mRNA pull-down assay was performed ${ }^{23}$ with minor modifications. Briefly, cells were harvested $24 \mathrm{~h}$ after transfection and lysed in lysis buffer (Cell Signaling, USA) containing $20 \times$ protease inhibitor (Roche) and 60 U RNaseOUT (Invitrogen). Protein A Dynabeads (Invitrogen, USA) and $2 \mu \mathrm{g}$ AGO-1-specific antibody were used for immunoprecipitation. The immunoprecipitate was treated with $20 \mu \mathrm{g} / \mathrm{ml}$ proteinase $\mathrm{K}$ for 10 $\mathrm{min}$ at $37^{\circ} \mathrm{C}$. RNA was extracted using the easy-BLUE kit (iNTRON, Korea), and cDNAs were synthesized with the SuperScript III First-Strand Synthesis System (Invitrogen). To determine if miR-9a directly bound CG11070, primers that amplified the fragments of its $3^{\prime}$-UTR that included the predicted miR- $9 a$ seed sequence matches were designed. Senseless and $s N P F R 1$ were used as positive controls and tubulin was used as a negative control (Supplementary Table 1).

Western blot. Briefly, 20 fly heads for each genotype were homogenized in RIPA buffer, and lysates were loaded in each lane of $10 \%$ SDS gels and transferred to nitrocellulose membrane. Membranes were blocked in 5\% BSA and incubated with primary antibodies at $4{ }^{\circ} \mathrm{C}$ overnight. After washing membranes with TBS-T, membranes were incubated with the appropriate secondary antibody. Using the ECL Western blotting detection reagent, membranes were developed and images were captured using FluorChem E image processor. Antibodies used were anti-Tau (1:1000, T46, Cat no. 13-6400, Invitrogen), anti-AT180 (1:1000, Cat no. MN1040, Invitrogen), anti-PHF-1 (1:1000, Cat no. MN1050, Invitrogen), anti-AT8 (1:1000, Cat no. MN1020, Invitrogen) and anti- $\beta$-actin (1:1000, Cat no. JLA20, DHSB). $\beta$ actin was used as a loading control. Signal intensity was quantified using ImageJ (NIH) software. Flies used were 30 days old after eclosion. For Western blot analysis of SH-SY5y cells, the following antibodies were used: anti-Tau (Cat no. ab64193, Abcam), anti- $\beta$-actin (Cat no. LF-PA0207, AB Frontier), anti-Myc (Cat no. C3956, Sigma), anti-HA (Cat no. H6908, Sigma), and anti-CHIP (Cat no. sc133066, Santa Cruz Biotechnology).

Immunoprecipitation. Cells were transfected with the indicated plasmids. After $24 \mathrm{~h}$ of transfection, cells were harvested and lysed with IP buffer (50 mM HEPES
pH 7.5, $150 \mathrm{mM} \mathrm{NaCl}, 1.5 \mathrm{mM} \mathrm{MgCl} 2,5 \mathrm{mM} \mathrm{KCl}, 0.1 \%$ Tween-20, $2 \mathrm{mM}$ DTT, and protease inhibitor cocktail (Roche)). Lysates were centrifuged at $9700 \times g$ for $30 \mathrm{~min}$ at $4^{\circ} \mathrm{C}$, and the collected supernatants were incubated with anti-HA agarose beads (Sigma) at $4{ }^{\circ} \mathrm{C}$ for $4 \mathrm{~h}$. The beads were then washed with buffer containing $50 \mathrm{mM}$ HEPES pH 7.5, $150 \mathrm{mM} \mathrm{NaCl}, 1.5 \mathrm{mM} \mathrm{MgCl}, 5 \mathrm{mM} \mathrm{KCl}$, $0.1 \%$ Tween- 20 , and $2 \mathrm{mM} \mathrm{DTT}$, and the bound proteins were eluted with $2 \times$ SDS sample buffer. Samples were quantified by Western blot after heating at $95^{\circ} \mathrm{C}$ for $10 \mathrm{~min}$.

His-ubiquitin pull-down assay. PCS2-His-ubiquitin was co-transfected with the indicated plasmids and after $24 \mathrm{~h}$ of transfection, transfected cells were treated with $10 \mu \mathrm{M}$ MG132 for an additional $6 \mathrm{~h}$. Cells were lysed in urea lysis buffer (8 M Urea, $0.3 \mathrm{M} \mathrm{NaCl}, 0.5 \mathrm{M} \mathrm{Na}_{2} \mathrm{HPO}_{4}, 0.05 \mathrm{M}$ Tris, $0.001 \mathrm{M}$ PMSF, $0.01 \mathrm{M}$ imidazole, $\mathrm{pH} 8.0$ ) and sonicated for $4 \mathrm{~min}$. Cell lysates were transferred to equilibrated Ni-NTA agarose and incubated for $4 \mathrm{~h}$ at room temperature. Beads were then washed five times with urea wash buffer $\left(8 \mathrm{M}\right.$ Urea, $0.3 \mathrm{M} \mathrm{NaCl}, 0.5 \mathrm{M} \mathrm{Na}_{2} \mathrm{HPO}_{4}, 0.05 \mathrm{M}$ Tris, $0.001 \mathrm{M}$ PMSF, $0.02 \mathrm{M}$ imidazole, $\mathrm{pH}$ 6.5) and conjugated proteins were eluted with $40 \mu \mathrm{L} 2 \mathrm{X} \mathrm{Laemmli} / \mathrm{Imidazole}(200 \mathrm{mM}$ imidazole). Eluted proteins were analyzed by Western blotting after heating the samples at $95^{\circ} \mathrm{C}$ for $10 \mathrm{~min}$.

Protein stability analysis. SH-SY5y cells were transfected with the indicated plasmids or siRNAs, and treated with $100 \mu \mathrm{g} / \mathrm{mL}$ cyclohexamide (CHX) or vehicle after $24 \mathrm{~h}$ of transfection. Cells were collected at the specified time points after CHX treatment and immunoblotted with antibodies against the specified proteins To evaluate autophagic degradation, Tau was co-transfected with STUB1 and $U B E 4 B$ for $24 \mathrm{~h}$, and cells were treated with $50 \mu \mathrm{M}$ Chloroquine or vehicle for $8 \mathrm{~h}$. Cells were collected and lysed, and lysates were immunoblotted with antibodies against the specified proteins.

In vivo confocal microscopy and image analyses. Immunofluorescence staining for anti-Tau 5 (1:200, ab3931, Abcam), anti-pTau AT8 (1:200, Cat no. MN1020, Invitrogen), anti-pTau AT180 (1:200, Cat no. MN1040, Invitrogen), anti-pTau PHF-1 (1:200, Cat no. MN1050, Invitrogen), anti-LC3 (1:200, Cat no. M152-3, MBL), anti-P62 (1:200, Cat no. PM045, MBL) and anti-Beclin (1:200, Cat no. PD017, MBL) was performed in Tau-BiFC mice models ${ }^{29}$. Fluorescence was observed by confocal microscopy (Nikon A1R, JAPAN). Pre-absorption with excess target protein or omission of primary antibody was used to demonstrate antibody specificity and remove background generated by the detection assay. Colocalization and quantitative assessment of images were conducted using NIH Image J v1.44 software.

Statistics and reproducibility. All experiments were performed more than three times. In case of animal study, ' $N$ ' represents the number of biologically independent animals. Boxplots were generated using the standard style except that the whiskers represent minimum to maximum. In bar charts, unless otherwise noted, data are presented as mean \pm SEM, and comparisons between groups were conducted using the Student's $t$-test considering $p<0.05$ to be statistically significant. For multiple comparisons, we performed a one-way analysis of variance followed by pairwise $t$-tests using the Bonferroni method to adjust the $p$-value threshold for significance. 
Reporting summary. Further information on research design is available in the Nature Research Reporting Summary linked to this article.

\section{Data availability}

The data that support the findings of this study are available from the corresponding author upon reasonable request. Source data are provided with this paper.

Received: 3 August 2020; Accepted: 29 April 2021;

Published online: 02 June 2021

\section{References}

1. Ballatore, C., Lee, V. M. \& Trojanowski, J. Q. Tau-mediated neurodegeneration in Alzheimer's disease and related disorders. Nat. Rev. Neurosci. 8, 663-672 (2007).

2. Haass, C. \& Selkoe, D. J. Soluble protein oligomers in neurodegeneration: lessons from the Alzheimer's amyloid beta-peptide. Nat. Rev. Mol. cell Biol. 8, 101-112 (2007).

3. Kadavath, $\mathrm{H}$. et al. Tau stabilizes microtubules by binding at the interface between tubulin heterodimers. Proc. Natl Acad. Sci. USA 112, 7501-7506 (2015).

4. Lee, V. M., Goedert, M. \& Trojanowski, J. Q. Neurodegenerative tauopathies. Annu. Rev. Neurosci. 24, 1121-1159 (2001).

5. Sarkar, S. Neurofibrillary tangles mediated human neuronal tauopathies: insights from fly models. J. Genet. 97, 783-793 (2018).

6. Sivanantharajah, L., Mudher, A. \& Shepherd, D. An evaluation of Drosophila as a model system for studying tauopathies such as Alzheimer's disease. J. Neurosci. Methods 319, 77-88 (2019).

7. Shulman, J. M. \& Feany, M. B. Genetic modifiers of tauopathy in Drosophila. Genetics 165, 1233-1242 (2003).

8. Kosmidis, S., Grammenoudi, S., Papanikolopoulou, K. \& Skoulakis, E. M Differential effects of Tau on the integrity and function of neurons essential for learning in Drosophila. J. Neurosci. 30, 464-477 (2010).

9. Chanu, S. I. \& Sarkar, S. Targeted downregulation of dMyc restricts neurofibrillary tangles mediated pathogenesis of human neuronal tauopathies in Drosophila. Biochimi biophys Acta Mol. Basis Dis. 1863, 2111-2119 (2017).

10. Wittmann, C. W. et al. Tauopathy in Drosophila: neurodegeneration without neurofibrillary tangles. Science 293, 711-714 (2001).

11. Bartel, D. P. MicroRNAs: target recognition and regulatory functions. Cell 136, 215-233 (2009).

12. Brodersen, P. \& Voinnet, O. Revisiting the principles of microRNA target recognition and mode of action. Nat. Rev. Mol. Cell Biol. 10, 141-148 (2009).

13. Edbauer, D. et al. Regulation of synaptic structure and function by FMRPassociated microRNAs miR-125b and miR-132. Neuron 65, 373-384 (2010).

14. Krutzfeldt, J. \& Stoffel, M. MicroRNAs: a new class of regulatory genes affecting metabolism. Cell Metab. 4, 9-12 (2006).

15. Lu, J. et al. MicroRNA expression profiles classify human cancers. Nature $\mathbf{4 3 5}$ 834-838 (2005).

16. Poy, M. N. et al. A pancreatic islet-specific microRNA regulates insulin secretion. Nature 432, 226-230 (2004).

17. Schaefer, A. et al. Cerebellar neurodegeneration in the absence of microRNAs. J. Exp. Med. 204, 1553-1558 (2007).

18. Goldberg, A. L. Protein degradation and protection against misfolded or damaged proteins. Nature 426, 895-899 (2003).

19. Keller, J. N., Hanni, K. B. \& Markesbery, W. R. Impaired proteasome function in Alzheimer's disease. J. neurochem. 75, 436-439 (2000).

20. Myeku, N. et al. Tau-driven 26S proteasome impairment and cognitive dysfunction can be prevented early in disease by activating cAMP-PKA signaling. Nat. Med. 22, 46-53 https://doi.org/doi:10.1038/nm.4011 (2016).

21. Petrucelli, L. et al. CHIP and Hsp70 regulate tau ubiquitination, degradation and aggregation. Hum. Mol. Genet. 13, 703-714 (2004).

22. Dolan, P. J. \& Johnson, G. V. A caspase cleaved form of tau is preferentially degraded through the autophagy pathway. J. Biol. Chem. 285, 21978-21987 (2010).

23. Suh, Y. S. et al. Genome-wide microRNA screening reveals that the evolutionary conserved miR-9a regulates body growth by targeting sNPFR1/ NPYR. Nat. Commun. 6, 7693 (2015).

24. Li, Y., Wang, F., Lee, J. A. \& Gao, F. B. MicroRNA-9a ensures the precise specification of sensory organ precursors in Drosophila. Genes Dev. 20, 2793-2805 (2006).

25. Lee, B. I. et al. Photoexcited Porphyrins as a Strong Suppressor of betaAmyloid Aggregation and Synaptic Toxicity. Angew. Chem. 54, 11472-11476 (2015).

26. Lee, B. I., Suh, Y. S., Chung, Y. J., Yu, K. \& Park, C. B. Shedding Light on Alzheimer's beta-Amyloidosis: Photosensitized Methylene Blue Inhibits Self-
Assembly of beta-Amyloid Peptides and Disintegrates Their Aggregates. Sci. Rep. 7, 7523 (2017).

27. Hatakeyama, S. et al. U-box protein carboxyl terminus of Hsc70-interacting protein (CHIP) mediates poly-ubiquitylation preferentially on four-repeat Tau and is involved in neurodegeneration of tauopathy. J. neurochemistry $\mathbf{9 1}$, 299-307 (2004).

28. Shimura, H., Schwartz, D., Gygi, S. P. \& Kosik, K. S. CHIP-Hsc70 complex ubiquitinates phosphorylated tau and enhances cell survival. J. Biol. Chem. 279, 4869-4876 (2004).

29. Shin, S. et al. Visualization of soluble tau oligomers in TauP301L-BiFC transgenic mice demonstrates the progression of tauopathy. Progress in neurobiology, 101782, https://doi.org/10.1016/j.pneurobio.2020.101782 (2020).

30. Berger, Z. et al. Rapamycin alleviates toxicity of different aggregate-prone proteins. Hum. Mol. Genet. 15, 433-442 (2006).

31. Sarkar, S., Davies, J. E., Huang, Z., Tunnacliffe, A. \& Rubinsztein, D. C. Trehalose, a novel mTOR-independent autophagy enhancer, accelerates the clearance of mutant huntingtin and alpha-synuclein. J. Biol. Chem. 282 5641-5652 (2007).

32. Chen, $X$. et al. Promoting tau secretion and propagation by hyperactive p300/ CBP via autophagy-lysosomal pathway in tauopathy. Mol. neurodegeneration 15, 2 (2020)

33. Hebert, S. S. \& De Strooper, B. Alterations of the microRNA network cause neurodegenerative disease. Trends Neurosci. 32, 199-206 (2009).

34. Wang, M., Qin, L. \& Tang, B. MicroRNAs in Alzheimer's Disease. Front. Genet. 10, 153 (2019).

35. Absalon, S., Kochanek, D. M., Raghavan, V. \& Krichevsky, A. M. MiR-26b, upregulated in Alzheimer's disease, activates cell cycle entry, tauphosphorylation, and apoptosis in postmitotic neurons. J. Neurosci.: Off. J. Soc Neurosci. 33, 14645-14659 (2013).

36. Dickson, J. R., Kruse, C., Montagna, D. R., Finsen, B. \& Wolfe, M. S. Alternative polyadenylation and miR-34 family members regulate tau expression. J. neurochemistry 127, 739-749 (2013).

37. Ma, X., Liu, L. \& Meng, J. MicroRNA-125b promotes neurons cell apoptosis and Tau phosphorylation in Alzheimer's disease. Neurosci. Lett. 661, 57-62 (2017).

38. Smith, P. Y. et al. miR-132/212 deficiency impairs tau metabolism and promotes pathological aggregation in vivo. Hum. Mol. Genet. 24, 6721-6735 (2015).

39. Wang, L. et al. Dihydrotanshinone I induced apoptosis and autophagy through caspase dependent pathway in colon cancer. Phytomedicine: Int. J. Phytother. phytopharmacology 22, 1079-1087 (2015).

40. Zhao, Z. B. et al. MicroRNA-922 promotes tau phosphorylation by downregulating ubiquitin carboxy-terminal hydrolase L1 (UCHL1) expression in the pathogenesis of Alzheimer's disease. Neuroscience 275, 232-237 (2014)

41. Coolen, M., Katz, S. \& Bally-Cuif, L. miR-9: a versatile regulator of neurogenesis. Front. Cell. Neurosci. 7, 220 (2013).

42. Delay, C., Mandemakers, W. \& Hebert, S. S. MicroRNAs in Alzheimer's disease. Neurobiol. Dis. 46, 285-290 (2012).

43. Kaneko, C. et al. Characterization of the mouse gene for the U-box-type ubiquitin ligase UFD2a. Biochemical biophysical Res. Commun. 300, 297-304 (2003).

44. Qiang, L. et al. Tau Does Not Stabilize Axonal Microtubules but Rather Enables Them to Have Long Labile Domains. Curr. Biol.: CB 28, 2181-2189 e2184 (2018).

45. Min, S. W. et al. Acetylation of tau inhibits its degradation and contributes to tauopathy. Neuron 67, 953-966 (2010).

46. Morris, M. et al. Tau post-translational modifications in wild-type and human amyloid precursor protein transgenic mice. Nat. Neurosci. 18, 1183-1189 (2015).

47. Wu, H. et al. UBE4B promotes Hdm2-mediated degradation of the tumor suppressor p53. Nat. Med. 17, 347-355 (2011).

48. Zeinab, R. A., Wu, H., Sergi, C. \& Leng, R. UBE4B: a promising regulatory molecule in neuronal death and survival. Int. J. Mol. Sci. 13, 16865-16879 (2012).

49. Matsumoto, M. et al. Molecular clearance of ataxin-3 is regulated by a mammalian E4. EMBO J. 23, 659-669 (2004).

50. Babu, J. R., Geetha, T. \& Wooten, M. W. Sequestosome 1/p62 shuttles polyubiquitinated tau for proteasomal degradation. J. neurochemistry $\mathbf{9 4}$, 192-203 (2005)

51. David, D. C. et al. Proteasomal degradation of tau protein. J. neurochemistry 83, 176-185 (2002).

52. Wong, E. S. et al. Autophagy-mediated clearance of aggresomes is not a universal phenomenon. Hum. Mol. Genet. 17, 2570-2582 (2008).

53. Schaeffer, V. et al. Stimulation of autophagy reduces neurodegeneration in a mouse model of human tauopathy. Brain: a J. Neurol. 135, 2169-2177 (2012).

54. Inoue, K. et al. Macroautophagy deficiency mediates age-dependent neurodegeneration through a phospho-tau pathway. Mol. neurodegeneration 7, 48 (2012)

55. Hamano, T. et al. Autophagic-lysosomal perturbation enhances tau aggregation in transfectants with induced wild-type tau expression. Eur. J. Neurosci. 27, 1119-1130 (2008) 
56. Wang, Y. et al. Synergy and antagonism of macroautophagy and chaperonemediated autophagy in a cell model of pathological tau aggregation. Autophagy 6, 182-183 (2010).

57. Kruger, U., Wang, Y., Kumar, S. \& Mandelkow, E. M. Autophagic degradation of tau in primary neurons and its enhancement by trehalose. Neurobiol. aging 33, 2291-2305 (2012).

58. Rodriguez-Navarro, J. A. et al. Trehalose ameliorates dopaminergic and tau pathology in parkin deleted/tau overexpressing mice through autophagy activation. Neurobiol. Dis. 39, 423-438 (2010).

59. Tan, J. M. et al. Lysine 63-linked ubiquitination promotes the formation and autophagic clearance of protein inclusions associated with neurodegenerative diseases. Hum. Mol. Genet. 17, 431-439 (2008).

60. Thrower, J. S., Hoffman, L., Rechsteiner, M. \& Pickart, C. M. Recognition of the polyubiquitin proteolytic signal. EMBO J. 19, 94-102 (2000).

61. Deng, Z. et al. Autophagy Receptors and Neurodegenerative Diseases. Trends cell Biol. 27, 491-504 (2017).

62. Lippai, M. \& Low, P. The role of the selective adaptor p62 and ubiquitin-like proteins in autophagy. BioMed. Res. Int. 2014, 832704 (2014).

63. Stolz, A., Ernst, A. \& Dikic, I. Cargo recognition and trafficking in selective autophagy. Nat. cell Biol. 16, 495-501 (2014).

64. Mohanty, S. et al. The E3/E4 ubiquitin conjugation factor UBE4B interacts with and ubiquitinates the HTLV-1 Tax oncoprotein to promote NF-kappaB activation. PLoS Pathog. 16, e1008504 (2020).

65. Tak, H. et al. Bimolecular fluorescence complementation; lighting-up tau-tau interaction in living cells. PloS one 8, e81682 (2013).

66. Lee, J. et al. Remodeling of heterochromatin structure slows neuropathological progression and prolongs survival in an animal model of Huntington's disease. Acta neuropathologica 134, 729-748 (2017).

\section{Acknowledgements}

Drosophila stocks were obtained from Bloomington Sock Center (Bloomington, IN, USA) and Vienna Drosophila RNAi Center (VDRC, Vienna, Austria). This work was supported by grants from KRIBB Research Initiative Program, National Research Council of Science \& Technology (CRC-15-04-KIST), KIST (2E30951, 2E30954 and 2E30962), and National Research Foundation of Korea (2015R1A5A1009024, 2017HID3A1A02054608, 2018M3C7A1056894, 2019R1A2C 2004052, 2019R1A2C2004149, 2020R1A4A4079494, and 2020M3E5D9079742).

\section{Author contributions}

M.S., E.J.S., H.R., and K.Y designed the research, M.S., S.J.H., T.D., and Y.S.S. performed experiments, M.S., Y.K.K., J.L., E.J.S., H.R., and K.Y. analyzed the data, and M.S., E.J.S., H.R., and K.Y. wrote the manuscript.

\section{Competing interests}

The authors declare no competing interests.

\section{Additional information}

Supplementary information The online version contains supplementary material available at https://doi.org/10.1038/s41467-021-23597-9.

Correspondence and requests for materials should be addressed to E.J.S., H.R. or K.Y.

Peer review information Nature Communications thanks Charbel Moussa and other, anonymous, reviewers for their contributions to the peer review of this work.

Reprints and permission information is available at http://www.nature.com/reprints

Publisher's note Springer Nature remains neutral with regard to jurisdictional claims in published maps and institutional affiliations.

(c) (i) Open Access This article is licensed under a Creative Commons Attribution 4.0 International License, which permits use, sharing, adaptation, distribution and reproduction in any medium or format, as long as you give appropriate credit to the original author(s) and the source, provide a link to the Creative Commons license, and indicate if changes were made. The images or other third party material in this article are included in the article's Creative Commons license, unless indicated otherwise in a credit line to the material. If material is not included in the article's Creative Commons license and your intended use is not permitted by statutory regulation or exceeds the permitted use, you will need to obtain permission directly from the copyright holder. To view a copy of this license, visit http://creativecommons.org/ licenses/by/4.0/.

(c) The Author(s) 2021, corrected publication 2021 